\title{
Die Geburt der Gegenkultur aus dem Geiste des Raketenstaates
}

\section{Zur Kunst der Prosa Thomas Pynchons}

Einer Randbemerkung in Ralf Simons Vorüberlegungen zur >Theorie der Prosa zufolge reicht deren literarhistorisch bedeutendste Tradition bis zur menippeischen Satire der Antike zurück. Das zentrale Argument dafür liefert die Herleitung von der lanx satura, die auf die >Formlosigkeit` bzw. Hybridität solcher Texte verweist. So stichhaltig diese Argumentation angesichts empirischer Beispiele von François Rabelais und Johann Fischart über Laurence Sterne und Jean Paul bis hin zu James Joyce und Arno Schmidt auch ist, die eine unbestritten »starke Affinität zu Satire, Humor und grotesker Verkörperung « aufweisen (Simon 2018, 416), so wirft sie aufgrund der enormen historischen Distanz, die zwischen Antike und Postmoderne liegt, doch auch Fragen auf. Neben der transhistorischen Perspektive betreffen diese insbesondere die literarische Originalität der jeweiligen Prosaprojekte, die sich im einfachen Rückgriff auf Gattungsmerkmale der Menippea nur unzulänglich erklären lässt. Grund genug, dieser Prosa in ihren literarhistorischen Bezügen genauer nachzuspüren.

Im Hinblick auf die Frage, »[w]as Prosa eigentlich sei« (Schlegel 1988, 211), erweist sich die Menippea als ein idealer Prüfstein, weil sie im Kontext des Kynismus entsteht und damit gemäß der antiken Konzeption der >Philosophie als Lebensform` (Hadot 1991) zwischen Theorie und Praxis changiert. Als Kulturtechnik stellt die Satire ein Schwellenphänomen dar, das über seine literarischen Verfahren nicht nur die Schnittstelle zwischen Philosophie und Lebenskunst prozessiert, sondern aufgrund ihrer praxeologischen Orientierung zugleich einen Beitrag zur Reflexion der Prosa zu leisten imstande ist. Entsprechend führt Ralf Simons (2013) Revision der Ästhetikgeschichte von Baumgarten bis Hegel zu einer Grundannahme, die alles andere als trivial ist, da sie dem distinktiven Merkmal des Elaborationsgrades Rechnung trägt, über den sich die Poetizität der Prosa näher bestimmen lässt. Denn letztlich ist es weniger die syntagmatische Gerichtetheit narrativer Elemente, die die Prosa auszeichnet, als ihre Stilistik oder Tonalität. Hierbei geht es um die Rhythmisierung der Sprache; um die Distribution von akzentuierten und nicht akzentuierten Silben, um syntaktische Akzentuierungen genauso wie semantische Akzente; es geht um den an der Mündlichkeit

Tobias Lachmann, Dortmund

Ә Open Access. (C 2021 Tobias Lachmann, publiziert von De Gruyter. (cc)BY Dieses Werk ist lizenziert unter einer Creative Commons Namensnennung 4.0 International Lizenz. https://doi.org/10.1515/9783110731569-008 
orientierten Fluss der Rede; um die Tonalität unterschiedlicher stilistischer Register; um die Selektion heterogenen sprachlichen Materials und dessen Mischung; zudem um Subjektsituationen und imaginäre Evokationen, die fremde Wahrnehmungswelten eröffnen; um innovative Darstellungsweisen für komplexe Theoreme, Weltanschauungen oder diskursive Positionen, die sich $\mathrm{zu}$ faszinierenden Texten vernetzen und im Medium der Literatur neue Erfahrungen ermöglichen. Kurz gesagt: Eine Theorie der Prosa lässt sich wohl nur auf dem Weg über diese Sprachkunst entwickeln, die ihrerseits auf das >prosaische` Leben reagiert. Durch Verfahren wie die genannten übernimmt die Prosa die Funktion, zwischen der lebensweltlichen Komplexität, die »vor den Formen der Kunst« liegt, und der literarischen Komplexität, die dahinter liegt, zu vermitteln (Simon 2013, 285). Sie verfügt über das komplette Spektrum literarischer Formen und Verfahren und unterzieht diese einer konsequenten ästhetischen Reflexion: „Die Analogie lautet dann: So wie die Wahrnehmung aus den Kanälen der fünf Sinne ein kompaktes, synästhetisch vermitteltes Percept macht, so macht die Prosa aus den Formen eine weitere Verdichtung, als formlose Form « (Simon 2013, 285). Deren Poetizität allerdings variiert in Abhängigkeit vom Maß an Elaboration der verwandten literarischen Verfahren. Durch eine Untersuchung der Regeln und Gesetzmäßigkeiten der Textgenese lässt sie sich genauer bestimmen.

Modellhaft betrachtet konstituiert sich die Prosa also im Zusammenspiel der drei Ebenen der aisthesis, der poiesis und der theoria, wobei der poiesis eine besondere Rolle zukommt: Sie umfasst die Darstellungsformen und den Herstellungsprozess gleichermaßen, sorgt auf der mittleren Ebene dafür, dass die vorgängige Wahrnehmung in der literarischen Praxis in Prosa überführt wird, während sie diese zugleich in den kontemplativen Raum der Schau stellt. Sie bringt Wissen, Wirklichkeit und Wahrheit hervor, anstatt in einem mimetischen Verhältnis zur Welt $\mathrm{zu}$ verharren. Wird dabei die Realismus-Frage virulent, so nicht etwa im Sinne der Abbildung oder Widerspiegelung einer vorgängigen Realität, sondern als Produktion von Wirklichkeit mit den Mitteln der Literatur, die auch pararealistische, also ins Phantastische reichende Spielarten umfassen können. Elaborierte Prosa zeichnet sich demnach dadurch aus, dass sie an Stelle des univektoriellen Widerspiegelungsmodells multivektorielle Bezüge zwischen Text und Welt etabliert und durch Applikationen andere Wirklichkeiten nicht bloß denkbar, sondern konkret werden lässt.

Auch wenn die hier skizzierte Genealogie dieser Traditionslinie keineswegs kontinuierlich gedacht werden darf, da sie von fundamentalen Diskontinuitäten gekennzeichnet ist, die sich an den epochalen Einschnitten der Antike, der Romantik und der Moderne bzw. Postmoderne exemplarisch festmachen lassen, erweist sich der antike Kynismus für die Kunst der Prosa als relevant. Unter dem Titel Der Mut zur Wahrheit hat sich Michel Foucault in seiner letzten Vorlesung 
am Collège de France eingehend mit der fragmentarisch überlieferten >Weisheit der Hunde auseinandergesetzt, die sich insbesondere durch ihre »Praxis der Verdiktion« (Foucault 2010, 207) auszeichnet. Darin reproduziert er das Bild des schmutzigen, nur mit dem Notwendigsten bewehrten Vagabunden, der sich am Rande der agorá aufhält, die Bürger der polis umgarnt, provoziert oder gar verspottet und sich ansonsten mit Betteleien durchbringt. Im entschiedenen Gegensatz $\mathrm{zu}$ den »Verhaltensregeln und kulturellen und gesellschaftlichen Werten in der Antike« (Foucault 2010, 246) pflegt der Kyniker eine Ästhetik der Existenz, die in einem ganz elementaren Sinn ımaterialistischı, da unmittelbar auf das bios, das sprichwörtlich nackte Leben, bezogen ist. Diesem Vorrang der Seinsweise über den schon in der Antike nur rudimentär ausgeprägten theoretischen Rahmen korrespondiert der kynische Imperativ zur permanenten Reflexion der Lebensformen. Frei von unnötigem Ballast begibt sich der Kyniker als kataskopos, als Avantgardist im doppelten Wortsinn, "vor die Front der Menschheit» und erkundet, "was dem Menschen in weltlichen Dingen zu- oder abträglich sein mag « (Foucault 2010, 220). Mit dieser Funktion sind die Merkmale der Heimatlosigkeit und des Nomadismus ebenso eng verbunden wie die auf diesem Wege zu gewinnende autarkeia, die neben der ataraxia als wichtigstes Ziel der antiken Philosophie gilt. Erreicht wird dieses Glück allerdings über ein Leben im Skandal, in dem der Kyniker seinen Mitmenschen durch sein schamloses Dasein sowohl praktisch als durch seine Lust am Streit auch theoretisch ihre Irrungen vor Augen führt. Dazu nutzt er das Prinzip der parrhesia und spricht in seiner franken und freien Rede schonungslos die Wahrheit aus. Theorie und Praxis überschneiden sich im Kynismus so weitgehend, dass er keiner Verbreitung durch eine elaborierte Lehre bedarf. Der Kynismus basiert einzig auf der Praxis des Wahrsprechens, „des Wahrsprechens ohne Scham und Furcht, des unbeschränkten und mutigen Wahrsprechens, des Wahrsprechens, das seinen Mut und seine Kühnheit so weit dehnt, daß daraus umgekehrt eine unerträgliche Unverfrorenheit wird« (Foucault 2010, 217 f.).

Wenn Foucault behauptet, Lebensweise und Wahrsprechen seien im Kynismus unmittelbar miteinander verbunden, dann lässt er allerdings die Medialität der Sprache außer Acht. Zwar konstatiert er, dass die Antike eine dezidiert »kynische Kunst und Literatur« kennt. Die Beobachtung, dass Satire und Komödie in dieser Zeit »von kynischen Themen durchzogen« sind und »zu einem gewissen Grad» einen nachgerade "privilegierten Ort für den Ausdruck kynischer Themen « darstellen, vernachlässigt jedoch, dass damit ganz spezifische Formen der Darstellung angesprochen sind, die sich in literaturwissenschaftlichen Begriffen genauer beschreiben lassen. Daraus folgt, dass sich die »Korrespondenz zwischen Lebensstil und Offenbarung der Wahrheit« (Foucault 2010, 246), die für den Kynismus so essentiell ist, in den Künsten wohl deshalb bis in die 
Moderne bzw. Postmoderne fortschreibt, weil sie in ihren materiellen wie symbolischen Praktiken »gegenüber der Kultur, den gesellschaftlichen Normen, den ästhetischen Werten und Kanons« eigene Darstellungsweisen entwickelt, die »eine polemische Beziehung der Reduktion, der Verweigerung und Aggression« sowie der »Entblößung des Elementaren der Existenz«, der »ständige[n] Ablehnung« und der »Verwerfung jeglicher schon gesicherten Form« beinhalten (Foucault 2010, 248).

Mit der Anekdote und der Menippea lassen sich in diesem Zusammenhang gleich zwei Formate hervorheben. So existiert eine Geschichte des Kynismus heute nur deshalb, weil dieser anekdotisch tradiert wird. Die Grundstruktur dazu liefert die apophthegmatische Anekdote mit der narrativen Trias von occasio, provocatio und dictum: "Als [Diogenes von Sinope] im Kraneion ein Sonnenbad nahm, trat Alexander [der Große] an ihn heran und sagte: >Erbitte von mir, was du willst`, worauf er antwortete: `Geh mir aus dem Licht!« (Steinmann 1999, 44). Charakteristisch für die kynische Haltung ist dabei das Moment der Inversion, mit dem der gesellschaftliche Außenseiter auf das mäzenatische Ansinnen der geschichtsträchtigen Figur reagiert. Hierbei fungiert das dictum gleich in doppelter Hinsicht als Provokation: als parrhesiastischer Akt sowie als Witz, in dem das närrische Gelächter desjenigen widerhallt, der im Spott über die herrschenden Zustände einer souveränen Widerständigkeit zum Ausdruck verhilft. Michael Niehaus (2013) nennt diese elementare Form die sprechende Anekdote, weil sie Mündlichkeit inszeniert; traditionell als pointierter Sprechakt einer historischen Figur, der die Situation transzendiert und etwas Typisches zum Ausdruck bringt. Daneben existiert die stumme Anekdote, die der elaborierten Prosa entgegenkommt, weil sie als minimaler, aber variabel ausbau- und anschlussfähiger narrativer Text ein privilegiertes Medium für Historiographie und Literatur gleichermaßen darstellt. Indem sie die fixen Grenzen des Apophthegmas überschreitet, sich der Erzählung von kleinen Ereignissen jenseits der >großen Geschichte، zuwendet und die fragmentarischen Splitter eines historischen Geschehens von geringer Wirkung, aber großer Signifikanz zu kaleidoskopischen Texturen montiert, vermag es die Prosa, sich das ästhetische Potential der Anekdote anzueignen, um im zwielichtigen Raum zwischen faktualem und fiktionalem Erzählen alternative Geschichtslandschaften zu simulieren.

Die Perspektive der generativen Poetik hilft, sich zu verdeutlichen, wie kleine Formen über Prozesse der diskursiven Integration in große Formen transformiert werden. Ganz ähnlich verfährt die menippeische Satire, die sich sämtliche Formen einzuverleiben vermag. Die dabei entscheidende Rolle des Kynismus hat niemand so klar formuliert wie Friedrich Nietzsche, der die diskursintegrativen Mechanismen im Kreativzyklus von elementarer und elaborierter Kultur der Antike in Die Geburt der Tragödie oder: Griechenthum und Pessimismus wie folgt beschreibt: 
Wenn die Tragödie alle früheren Kunstgattungen in sich aufgesaugt hatte, so darf dasselbe wiederum in einem excentrischen Sinne vom platonischen Dialoge gelten, der, durch Mischung aller vorhandenen Stile und Formen erzeugt, zwischen Erzählung, Lyrik, Drama, zwischen Prosa und Poesie in der Mitte schwebt und damit auch das strenge ältere Gesetz der einheitlichen sprachlichen Form durchbrochen hat; auf welchem Wege die cynischen Schriften noch weiter gegangen sind, die in der grössten Buntscheckigkeit des Stils, im Hin- und Herschwanken zwischen prosaischen und metrischen Formen auch das litterarische Bild des "rasenden Sokrates«, den sie im Leben darzustellen pflegten, erreicht haben.

(Nietzsche 1972, 89)

Neben dem Bruch etablierter Konventionen hebt Nietzsche die Hybridisierung sowie Figuren der Steigerung und Überbietung besonders hervor. In der »Buntscheckigkeit des Stils« der Menippea findet die kynische Kongruenz von Philosophie und Lebensweise ihre literarische Entsprechung. Das auf dem Ernsthaft-Komischen (spoudogeloion) beruhende, 'proteischer Konzept der Menippea dient der Prosa insofern als Modell, als es eine »außerordentliche Freiheit in der Erfindung des Sujets und der philosophischen Idee« (Bachtin 1985, 127) genießt, die es gewissermaßen spielerisch erlaubt, Prosa im Paradoxon jener sformlosen Form zu generieren, die sich kontinuierlich selbst reflektiert, variiert und aktualisiert, permanent im Werden und daher nie mit sich selbst identisch ist. Die Interdiskursivität fungiert dabei als entscheidendes generatives Subsystem. Strukturell basiert die menippeische Satire auf Charakteristika, die Bachtin zufolge aus dem »transformierenden Einfluß des karnevalistischen Weltempfindens « (Bachtin 1985, 120) hervorgehen. Diese elementaren Merkmale lassen sich wie folgt verschlagworten: Erstens als Kairologie, die auf ihr »Verhältnis zur Wirklichkeit« zielt und die »aktuelle Gegenwart« zum »Ausgangspunkt für das Verständnis, die Beurteilung und die Darstellung der Wirklichkeit« (Bachtin 1985, 120) macht. Julia Kristeva spricht von einer »Art politische[m] Journalismus der Zeit«, in dem sich aktuelle politische Konflikte diskursiv manifestieren (Kristeva 1972, 368). Zweitens als Ereignishaftigkeit, denn sie "stützen sich nicht auf Überlieferung und widmen sich ihr nicht; sie stützen sich bewußt auf (allerdings noch nicht hinreichend gereifte) Erfahrung und freie Erfindung; ihr Verhältnis zur Überlieferung ist in der Mehrzahl der Fälle äußerst kritisch, bisweilen zynisch-entlarvend « (Bachtin 1985, 120). Und drittens als über die von Bachtin und Kristeva konstatierte Dialogizität und Intertextualität hinausgehende Interdiskursivität, die jene »intendierte Vielzahl der Stile und verschiedenen Stimmen« (Bachtin 1985, 121) umfasst:

Für sie ist eine Vielzahl der Töne in der Erzählung, die Vermischung des Hohen mit dem Niedrigen, des Ernsten mit dem Komischen charakteristisch, sie fügen in großem Ausmaß andere Gattungen ein - Briefe, gefundene Manuskripte, nacherzählte Dialoge, Parodien auf Gattungen der hohen Literatur, parodistisch umgedeutete Zitate $\mathrm{u}$. a.; in einigen von 
ihnen wird Prosa- und Verssprache vermischt, werden lebende Dialekte und Jargons verwendet (in der römischen Zeit auch direkte Zweisprachigkeit), zeigt sich der Autor in verschiedenen Masken. [...] Ein radikal neues Verhältnis zum Wort als dem Material der Literatur wird sichtbar.

(Bachtin 1985, 121)

Stets bleibt sie dabei dem spoudogeloion verpflichtet. Allerdings potenziert sie das Ernsthaft-Komische durch die verschiedenen Verfahren des Witzes bis hin zur satirischen Attacke und entfaltet auf diese Weise auch ihre politische Dimension: "Durch den Status ihrer Wörter ist sie politisch und gesellschaftlich subversiv. Sie befreit die Rede vom historischen Zwang, was eine absolute Kühnheit der philosophischen Intervention und Einbildungskraft mit sich bringt « (Kristeva 1972, 366). Dieser Aspekt ist in der Forschung zur Menippea häufig übersehen worden, da Bachtins Formulierung von der »Atmosphäre fröhlicher Relativität " (Bachtin 1985, 119) dazu verleitet, über die aggressive Tendenz der Satire hinwegzusehen, die "auf verbale und damit soziale Vernichtung des satirischen Opfers « aus ist: „Die Satire gibt [...] nicht nur eine alternative und komplementäre Weltsicht, sondern sie wendet sich auch aggressiv und entlarvend gegen angemaßte Autorität, gegen lebensbedrohende Ordnungen und falsche Normen « (Weiß 1985, 246). Entschieden bezieht sie die Position der sozialen Randständigkeit und der Gegenkultur. Darüber hinaus liefert sie auch für die ebenso eigentümliche wie sparasitäre` Formlosigkeit der Prosa, die zugleich alle Formen in sich zu versammeln weiß, das Modell: »Die Bewegung, die zwischen den beiden Polen, die der Dialog [zweier Diskurse] voraussetzt, entsteht, merzt aus unserem philosophischen Raum die Probleme der Kausalität, der Finalität usw. aus und deutet den Wert des dialogischen Prinzips für einen Denkraum an, der viel weiter wäre als der romanhafte« (Kristeva 1972, 374). Genau darin läge, meiner Ansicht nach, schließlich auch das einzigartige Potential der Prosa als Denkraum auf der Ebene der theoria.

In literarhistorischer Sicht bleibt zu bekräftigen, dass selbstverständlich nicht von einer einfachen Identität zwischen antiker Satire und postmoderner Prosa auszugehen ist. Sehr wohl lassen sich aber Anhaltspunkte finden, um die These der geteilten transhistorischen Dimension von Satire und Prosa zu untermauern. So erfährt die Prosa in der Romantik einen entscheidenden Modernisierungsschub, indem sie - wie bei August Wilhelm und Friedrich Schlegel - als kulturrevolutionäre Diskursbastelei gefasst wird. Am 116. Athenäums-Fragment lässt sich das Verfahren der radikalisierten Interdiskursivität gut festmachen (Schlegel 1967, 182f.): Wenn Friedrich Schlegel die »romantische Poesie« dort als "progressive Universalpoesie« definiert, deren zentrale Funktion es ist, über die etablierten literarischen Gattungen hinaus »Poesie und Prosa, Genialität und Kritik, Kunstpoesie und Naturpoesie« zu »mischen« oder gar zu »verschmelzen«, dann ist nicht zu übersehen, dass die diskursive Hybridisierung das zen- 
trale Element seines Konzepts ausmacht. Wird damit überdies das Ziel verknüpft, »die Poesie lebendig und gesellig, und das Leben und die Gesellschaft poetisch [zu] machen, den Witz [zu] poetisieren, und die Formen der Kunst mit gediegnem Bildungsstoff jeder Art an[zu]füllen und [zu] sättigen, und durch die Schwingungen des Humors [zu] beseelen«, dann zeigt sich, dass dies nur über Deterritorialisierungen, vor allem zwischen aisthesis, poiesis und theoria, geschehen kann. Die angestrebte Aufhebung der Trennung zwischen U- und E-Kultur, wie wir heute sagen würden, kommt darin zum Ausdruck, dass mit dem Alltäglichen das Prosaische und mit dem Populären die Gesamtheit aller die Gesellschaft betreffenden Bereiche angesprochen wird. Interessanterweise schließt Schlegels Auffassung auch das Vermögen ein, die Subjektivität des Autors als interdiskursiv programmiert zu denken und diskursive Positionen in ihrer Vielfalt zu inszenieren. Dadurch erhebt die Prosa den Anspruch, »ein Spiegel der ganzen umgebenden Welt, ein Bild des Zeitalters [zu] werden«. Wenig überraschend fungiert die poiesis dabei als zentrale Dimension. Denn Schlegel ist sich bewusst, dass ein elaborierter Text nur aus elementaren Bauteilen zu generieren ist. Das eigentliche Prinzip der Prosa ist daher der offene künstlerische Prozess, das stetige Werden:

Die romantische Poesie ist unter den Künsten was der Witz der Philosophie, und die Gesellschaft, Umgang, Freundschaft und Liebe im Leben ist. Andre Dichtarten sind fertig, und können nun vollständig zergliedert werden. Die romantische Dichtart ist noch im Werden; ja das ist ihr eigentliches Wesen, daß sie ewig nur werden, nie vollendet sein kann. Sie kann durch keine Theorie erschöpft werden, und nur eine divinatorische Kritik dürfte es wagen, ihr Ideal charakterisieren zu wollen. Sie allein ist unendlich, wie sie allein frei ist, und das als ihr erstes Gesetz anerkennt, daß die Willkür des Dichters kein Gesetz über sich leide.

(Schlegel 1967, 182f.)

Übrigens ist es ein oft übersehener Umstand, dass der Kynismus - und vor allem dessen »Oszillieren [...] zwischen Moral und Witz« (Niehues-Pröbsting 1988, 300) - für Schlegel den zentralen theoretischen Bezugspunkt darstellt. Seinem Talent zur produktiven Entwendung entsprechend, entwickelt er in der Auseinandersetzung mit dem Kynismus »eine universelle Form von Intellektualität, die er nicht auf die historisch so genannten Kyniker beschränkte und in der er wesentliche der eigenen intellektuellen Intentionen glaubte wiederzufinden« (Niehues-Pröbsting 1988, 300). Eine vergleichbare Beobachtung macht im neunzehnten Jahrhundert Rudolf Haym, der über Schlegel schreibt: "»Litterarischer Cynismus`, das wird jetzt die Formel für seine Existenzweise, den Cynismus überhaupt stempelt er zu seinem Lebensideal« (Haym 1870, 242). Als Beleg für diese Sichtweise sei hier beispielhaft für die unzähligen Notizen Schlegels das 851. Fragment zur Philosophie zitiert, aus dem die zentrale Funktion der kritischen Kulturtechnik des Kynismus eindeutig hervorgeht: 
Witz ist ein wesent[licher] Bestandtheil d[es] Cynismus, aber nur d[es] polemischen und naiven. Der Cyniker verachtet die Kunst $\pi[$ poesie] | und Kunst $\varphi$ [philosophie], hat aber Na$\operatorname{tur} \pi[$ poesie] $<$ Natur $\varphi$ [philosophie] $>$; Ironie ist nicht in ihm. Cynismus ist gar nicht auf $\eta \vartheta$ [Ethik] eingeschränkt. - Cyn.[ismus] ist moralische Genialität. - (Schlegel 1963, 100)

Inwiefern sich der literarische Kynismus über die Romantik hinaus bis in die Postmoderne weiterentwickelt, soll im Folgenden anhand eines Beispiels diskutiert werden, das als ein prototypischer postmoderner Text gilt und die literarische Welt bei seiner Veröffentlichung zutiefst gespalten und verstört hat: Thomas Pynchons Gravity's Rainbow (1973). Diesem Text, nach Elfriede Jelinek zu Deutsch Die Enden der Parabel (1981), haftet noch immer der Ruf eines Skandalbuches an. $\mathrm{Zu}$ hartnäckig hält sich das Urteil jenes advisory boards, das sich 1974 dazu entschloss, in der Kategorie fiction lieber gar keinen Pulitzer-Preis zu vergeben als diesen Pynchon zu verleihen, dessen nominierten Text man als »obscene«, »unreadable«, »turgid« und »overwritten« kritisierte (Kihss 1974, 38). Diese Begründung ist insofern interessant, als sowohl das Oxford English Dictionary als auch das amerikanische Merriam-Webster belegen, dass sich das intransitive Verb (to) overwrite auf die Stilistik eines Textes bezieht und dabei eine Schreibweise moniert, die als "too elaborately or ornately« geschrieben wahrgenommen wird. Beide Monita, der hohe Grad an Elaboration genauso wie die Rhetorisierung der Sprache, jedoch sind zentrale Charakteristika jener Prosa, deren Kunst hier auf den Grund gegangen werden soll. Paradoxerweise spricht die ablehnende JuryEntscheidung also doch für den Text, bei dem es sich Theodore D. Khapertian (1990) zufolge um eine menippeische Satire handelt. Daher soll im Folgenden das Experiment gewagt werden, auf Grundlage einer generativen Diskursanalyse (Link 1983) zu zeigen, wie die Prosa komplexe Wissensbereiche fasst, mithilfe ihrer Darstellungsoptionen formatiert und als Erkenntnisobjekte konstituiert. Die Dimension der poiesis rückt dabei ins Zentrum der Betrachtung.

\section{1 aisthesis als kynische Praxis: Pynchons Schreibszene und Poetik im Elementardiskurs}

Thomas Pynchon gilt noch immer als `Phantom der Literatur`. Tatsächlich ist relativ wenig über seine Schreibszene (Stingelin 2004, Giuriato et al. 2005 und Giuriato et al. 2006) bekannt und das, was man weiß, muss in der Forschungsliteratur erst aufgefunden werden. So profitiert Pynchon den Berichten seiner Verleger und Lektoren zufolge bei der Arbeit an Gravity's Rainbow von den umfassenden Beständen der Universitätsbibliothek der University of California in Berkeley, recherchiert während der langjährigen Textgenese aber auch andern- 
orts, etwa in Berlin. Seinem Freund Jules Siegel verdanken wir die Information, dass Pynchon in dieser Zeit zumeist auf mathematischem Millimeterpapier schreibt, das sich in seinem Apartment in Manhattan Beach - »a monk's cell decorated by the Salvation Army“ (Siegel 1977, 172) - zu einem dicken Stapel dieses mit seiner feinen, Druck- und Schreibschrift kombinierenden Handschrift überzogenen, quadrillierten Papiers schichtet: „the draft of Gravity's Rainbow, which he was in the process of typing and rewriting«. Da ein Großteil des Entwurfs während Pynchons Zeit in Mexiko und dort wohl nicht ohne den stimulierenden Einfluss von Cannabis entsteht, plagt sich der Autor, der die Arbeit am Manuskript von Gravity's Rainbow aus Geldsorgen für eine schnelle Veröffentlichung von The Crying of Lot 49 (1966) unterbrochen hat, an seiner Olivetti-Reiseschreibmaschine beim Versuch, den Text zu rekonstruieren: "'I was so fucked up while I was writing it, ‘ he said, sthat now I go back over some of those sequences and I can't figure out what I could have meant« (Siegel 1977, 172).

Da es Pynchon bevorzugt, seine Texte für sich sprechen zu lassen, sind Aussagen zu seiner Poetik rar gesät. Eine Ausnahme bildet der Band Slow Learner (1984), der Pynchons frühe Kurzprosa versammelt und vom Autor mit einem Vorwort versehen wird. Darin stellt er sich als Zaungast der Beatbewegung dar, der er ambivalent gegenübersteht. Einerseits zeigt er sich fasziniert von den kulturellen Innovationen der Beatniks, andererseits ist er sich darüber im Klaren, der Bewegung aufgrund seines familiären Hintergrundes sozial nicht zuzugehören: „Wir befanden uns in einer Übergangsphase, einer merkwürdigen kulturellen Wendezeit im Windschatten der Beats, und unsere Loyalität war tief gespalten« (Pynchon 1985, 16). Diese Wahrnehmung geht auf eine Verkettung zeitgenössischer Kontroversen zurück, in der sich soziale Konflikte mit generationellen, sprachlichen und kulturellen verknüpfen, auf das eigene Selbstverständnis zielen und eine wie auch immer geartete Subjektivierung jenseits der gängigen gesellschaftlichen Positionen erforderlich machen. Das betrifft Fragen der Stratifikation, also Klassenaspekte wie die vielfältigen Oppositionen zwischen Studenten und Arbeiterschaft, Fragen der Identifikation mit den Ausdrucksformen der klassischen Moderne oder den sprachlichen Experimenten der Beat-Autoren, sowie drängende Fragen nach alternativen Literatur- und Lebenskonzepten, die es erst noch zu entwickeln gilt; was bedeutet, »die Posen und Requisiten der Beat-Bewegung zu übernehmen und«, so Pynchon, »als Post-Beatniks, zu einem tieferen Verständnis dessen zu gelangen, was letztlich nichts anderes war als eine nüchterne und glaubwürdige Bekräftigung der Ideale, die wir alle für die eigentlich amerikanischen halten möchten« (Pynchon 1985, 16).

Literarischen Ausdruck findet diese Standortbestimmung in Entropy (1960), einem kurzen Prosatext, in dem der poeta doctus probiert, »den Stories der Beats so nahezukommen, wie es mir damals nur möglich war - obwohl ich na- 
türlich glaubte, dem Beatnik-Feeling mit angelesener Naturwissenschaft intellektuellen Glanz zu verleihen« (Pynchon 1985, 22). Das eigentliche Problem liegt denn auch in der Suche nach einer ebenso authentischen wie gesellschaftspolitisch relevanten literarischen Position jenseits der Dichotomie von underdogs und Arrivierten. Dass die akademische Tradition der Ivy League Colleges eine solche nicht bietet, zeigen die Beatniks deutlich auf: »[D]ank all der alternativen Nachrichten von unten, die durch den Efeu hereinsickerten, begannen wir ein Gespür für jene andere Welt zu entwickeln, die dort draußen summte und vibrierte« (Pynchon 1985, 15). Angeregt von Helen Waddells wiederaufgelegter Studie über die mittelalterliche Vagantendichtung beginnt Pynchon, während er Gravity’s Rainbow schreibt, eine eigenständige literarische Praxis zu entwickeln:

Diese Empfindung der Beengtheit im akademischen Elfenbeinturm trug sicher zu dem verführerischen Reiz des pikaresken amerikanischen Wanderlebens bei, das uns die Beat-Autoren zu führen schienen. Lehrlinge sind in allen Zünften und zu allen Zeiten begierig gewesen, auf die Wanderschaft zu gehen. [...] Ich hatte einen Roman veröffentlicht und glaubte, das ein oder andere zu wissen; vor allem aber scheint mir, daß ich es damals schaffte, mein Maul zu halten und den amerikanischen Stimmen zuzuhören, die mich umgaben. Es gelang mir sogar, meinen Blick von den gedruckten Quellen loszureißen und auf die amerikanische Wirklichkeit zu richten, wie sie sich außerhalb der Buchdeckel abspielt. Ich war endlich on the road, kam selbst an die Orte, von denen Kerouac geschrieben hatte.

(Pynchon 1985, 32)

Es ist eine bemerkenswerte historische Diskontinuität, die dazu führt, dass Pynchon auf dem Umweg über seine Waddell-Rezeption auf die Parallelen zwischen Beatniks und Goliarden stößt, die als Wanderprediger die im Mittelalter nur schwer zu vereinbarenden Merkmale der akademischen Bildung und der Volkstümlichkeit verkörpern. Vielsagend ist dieser Umstand auch deshalb, weil in den Bettelorden des Mittelalters auch der Kynismus tradiert wird; und zwar von Menschen, »die auf alles verzichten, die einfachsten und grobschlächtigsten Kleider tragen und barfuß umherziehen, um die Menschen aufzurufen, sich um ihr Heil zu kümmern, und die sie in ihren Schmähreden anfahren, deren Heftigkeit bekannt ist« (Foucault 2010, 240). Ähnlich wie Foucault weiß Kristeva davon $\mathrm{zu}$ berichten, dass »der menippeische Aspekt« im Mittelalter »von der Autorität des religiösen Textes bezwungen [wird] und während der bürgerlichen Ära vom Absolutismus des Individuums und der Dinge« (Kristeva 1972, 370). Allerdings produziert diese Verdrängungsbewegung mit der Vagantendichtung zugleich eine ambulante Literatur, in der die menippeische Tradition in den nomadischen Diskursformen der umherziehenden Scholaren bewahrt werden kann. In diesem Sinne betont Waddell am »historical interest of the Vagantes as one of the earliest disintegrating forces in the medieval church«, dass deren literarhistorischer Platz vor allem »in the development of satire and the secularising of the stage» 
(Waddell 1980, v) bestehe. Pynchon bestätigt diese Sichtweise im Vorwort seines Kurzprosa-Bandes, wenn er sie neben der Beat-Bewegung als eine zweite, historische Inspirationsquelle für sein Schreibprojekt ins Spiel bringt:

Einen verstärkenden Effekt übte, jedenfalls auf mich, Helen Waddels [sic!] The Wandering Scholars aus, eine in den frühen 50er Jahren wiederaufgelegte Studie über die Vagantendichter des Mittelalters, die in einem großen Aufbruch die Klöster mit den Straßen Europas vertauschten und in ihren Liedern die Vielfalt des Lebens feierten, die sie außerhalb der akademischen Mauern fanden. Angesichts meiner damaligen Universitäts-Umgebung waren die Parallelen nicht schwer zu sehen.

(Pynchon 1985, 14f.)

Aus seiner eigenen nomadisierenden Daseinsweise geht mit Gravity's Rainbow schließlich ein Prosatext hervor, der trotz seines historisch auf die Zeit gegen Ende des Zweiten Weltkrieges konzentrierten Sujets zugleich unmittelbar auf seine Entstehungszeit zwischen 1963 und 1973 bezogen ist und daher nicht zu Unrecht als zentraler Text der literarischen Postmoderne gilt. Die kairologische Dimension dieses Schreibprojekts manifestiert sich vor allem in seiner elaborierten Darstellungsweise, die es Pynchon in einem literarischen Coup ermöglicht, eine gegen Ende des Zweiten Weltkriegs abgefeuerte deutsche V2-Rakete - »A screaming comes across the sky“ (Pynchon 1973, 3) - in das Orpheus-Filmtheater in Los Angeles einschlagen zu lassen, das er durch das kryptographische Verfahren der polyalphabetischen Substitution der Grapheme >m` und >s genauso generiert, wie er es durch den intertextuellen Verweis auf Ovids Metamorphosen fiktionalisiert, um damit die Gegenwärtigkeit der Geschichte spektakulär unter Beweis zu stellen. Schließlich wird seit dem 6. August 1945 nicht nur der literarhistorische Moment von der Atombombe definiert, die von Langstreckenraketen, wie sie im Rahmen des Hermes Missile Program in White Sands erprobt werden, binnen kürzester Zeit in alle Teile der Welt getragen werden können:

Wir alle haben auf diese schleichende Eskalation unsrer Hilflosigkeit und unseres Schreckens mit den wenigen Mitteln reagiert, die uns zur Verfügung stehen, vom Versuch, nicht darüber nachzudenken, bis zur Flucht in den Wahnsinn. Irgendwo auf diesem Spektrum der Machtlosigkeit befindet sich das Schreiben von Literatur darüber. (Pynchon 1985, 28)

So spärlich diese poetologischen Aussagen auch erscheinen mögen, genau besehen vermitteln sie doch eine relativ konkrete Vorstellung davon, worum es in Pynchons Prosaprojekt geht: um eine ungebundene Existenzweise, ein Leben ganz im Zeichen der Literatur und eine provokative Entgrenzung der Oppositionen zwischen klassischer Moderne und Beat, zwischen Bildungselite und seinfachem`Volk, zwischen Ivy League und the street. Darüber hinaus ist Pynchon aufgrund seiner Ausbildung imstande, etwas zu leisten, was nur die Wenigsten vermögen: Er vermag es, die Kluft zwischen den `zwei Kulturen`, zwischen der literarischen und der naturwissenschaftlichen Intelligenz (Snow 1987), zu über- 
brücken. Denn als in das US-amerikanische Minuteman Program involvierter ehemaliger Redakteur des Bomarc Aero-Space Department bei der Boeing Airplane Co. in Seattle (Pynchon 2003) ist der studierte Ingenieur im Besitz eines dermaßen exakten Wissens über die technische Welt, dass mancher literarisch ambitionierte Physiker vor Neid erblassen würde. Diese Konstellation führt auch dazu, dass Pynchons technohistorisches Interesse stets an den politischen und ideologischen Konflikten der Gegenwart orientiert bleibt. In seinem Essay Is It $O$. K. To Be A Luddite? (1984) zeigt er auf, wohin der naive Fortschrittsoptimismus der Moderne geführt hat:

By 1945, the factory system - which, more than any piece of machinery, was the real and major result of the Industrial Revolution - had been extended to include the Manhattan Project, the German long-range rocket program and the death camps, such as Auschwitz. It has taken no major gift of prophecy to see how these three curves of development might plausibly converge, and before too long. Since Hiroshima, we have watched nuclear weapons multiply out of control, and delivery systems acquire, for global purposes, unlimited range and accuracy. An unblinking acceptance of a holocaust running to seven- and eight-figure body counts has become - among those who, particularly since 1980, have been guiding our military policies - conventional wisdom.

(Pynchon 1984, 41)

Damit stellt sich die drängende Frage nach der Rolle kritischer Intellektueller, die aufgrund ihrer umfassenden Bildung in der Lage sind, das sich immer weiter differenzierende Spektrum des spezialisierten Wissens moderner Gesellschaften zu entdifferenzieren, $\mathrm{zu}$ integrieren und subjektiv applizierbar $\mathrm{zu}$ machen. Dabei geht es nicht um l'art pour l'art, sondern um eine Literatur, die es vermag, aufgrund ihrer Darstellungsformen einer globalen Realität gerecht zu werden, die von einer wachsenden Komplexität gekennzeichnet und von einer fein ziselierten Mikrophysik der Macht durchzogen ist.

As well-known President and unintentional Luddite D.D. Eisenhower prophesied when he left office, there is now a permanent power establishment of admirals, generals and corporate CEO's, up against whom us average poor bastards are completely outclassed, although Ike didn't put it quite that way. We are all supposed to keep tranquil and allow it to go on, even though, because of the data revolution, it becomes every day less possible to fool any of the people any of the time.

(Pynchon 1984, 41)

Wie also lässt sich der >Wahrheit der technischen Weltı ins Auge blicken, während man zugleich versucht, das im Computerzeitalter ebenso allgegenwärtige wie leicht verfügbare Wissen gegen die Machtverhältnisse $\mathrm{zu}$ wenden? Im Essay führt Pynchon es vor, indem er historische Fakten zur Industriellen Revolution und zum Aufkommen des Luddismus mit begriffsgeschichtlichen Erläuterungen sowie enzyklopädisch dokumentierten Erzählungen über den Volkshelden Ned Ludd verknüpft, die er mit der Technikgeschichte der Entwicklung des 
Webstuhls ebenso eng führt wie mit der Sozialgeschichte des entstehenden Klassenkampfes, den er im Sinne des Historischen Materialismus kontextualisiert, ohne dabei auf den >German philosopher` zu rekurrieren, da die Effekte der wachsenden Arbeitslosigkeit bei gleichzeitiger Akkumulation von Kapital schon im achtzehnten Jahrhundert für jedermann offensichtlich sind. Vor allem aber öffnet er einen literarhistorischen Blick auf bekannte Stoffe der populären Literatur wie Mary Shelleys Frankenstein; or, The Modern Prometheus (1818), inklusive der Verfilmung von James Whale, Horace Walpoles The Castle of Otranto (1764) oder King Kong und die weiße Frau (1933), die sich aus Sicht der Maschinenstürmer weniger als simple Science Fiction, Horror- oder Monstergeschichten darstellen, als sie eine Gemeinsamkeit teilen, die darin besteht, dass sie in ihren Genrekonventionen die menschliche Hybris im Umgang mit der Natur, das Eigenleben von Technologien und die Möglichkeit der Verselbstständigung der technologischen Entwicklung thematisieren. Diese Sichtweise erscheint bemerkenswert, weil sie erkennbar von der oberflächlichen Rezeption der genannten Texte abweicht und diesen eine über die bloße `Effekthascherei` des Horrors hinausweisende analytische Kapazität zugesteht, die auf die Gouvernementalität ihrer jeweiligen Entstehungszeit zielt. Analytisch im Hinblick auf die Gegebenheiten der >wirklichen jedoch nicht allein, weil sie sich in der ebenso ungezwungenen wie schlichten Rede alltäglicher Kommunikation auf die Wirklichkeit beziehen und insofern als >prosaisch zu bezeichnen wären. Vielmehr fällt auf, dass alle zitierten Beispiele mit phantastischen Elementen arbeiten und damit die Grenzen des Denkbaren verschieben, auch wenn dies bedeuten mag, entschieden gegen die vermeintlichen Gesetze einer realistischen Darstellung zu verstoßen. Wie der Autor eingesteht, sehen sie sich daher dem Vorbehalt mangelnder Ernsthaftigkeit ausgesetzt: "But if we do insist upon fictional violations of the laws of nature - of space, time, thermodynamics, and the big one, mortality itself - then we risk being judged by the literary mainstream as Insufficiently Serious « (Pynchon 1984, 41). Es hat jedoch den Anschein, als wolle Pynchon - nur aufgrund des unterstellten höheren Unsinns - keineswegs von seiner Überzeugung abrücken, dass eine kunstvolle Vermischung von Faktischem und Fiktionalem allein dazu imstande ist, die komplexen Zusammenhänge zu erhellen. Wenn er also davon spricht, dass er >das Schreiben von Literatur` als Strategie im Umgang mit der politischen Lage der Nachkriegszeit begreift, dann geht er die konstatierte Hilflosigkeit und den Schrecken des Atomzeitalters produktiv an. Dies allerdings nicht agitatorisch, sondern ästhetisch. Schließlich entfaltet Pynchon in der Arbeit an Gravity's Rainbow das volle Potential seiner Prosa, die sich weniger als Erzählform denn als Erkenntnismedium begreifen lässt. 


\section{2 poiesis: Gravity's Rainbow als kynische Prosa im Interdiskurs}

Konzentriert man sich nach diesen allenfalls rudimentären Ausführungen zu den externen Rahmenbedingungen von Autorschaft, Poetik und Schreibszene nun auf die Vermittlung von Dingen und Worten in Pynchons Prosa, dann fällt auf, dass der historische Prozess als komplexes Geflecht aus Handlungen und Ereignissen in Gravity's Rainbow eine zentrale Instanz zur Strukturierung der literarischen Textur liefert. Überall tauchen historisch verbürgte Figuren auf, zum Teil sogar große Figuren der Zeitgeschichte. Wieder und wieder bezieht sich der Text, mal explizit, mal andeutungsweise, auf bedeutende historische Daten. Das Problem daran liegt in dem Umstand, dass die Rede von >Geschichte`, ganz gleich, ob man sie nun als Historiographie oder als erzählenden Text im Feld der Literatur versteht, leicht dazu verleitet, von einer narrativen Gerichtetheit auf ein Erzählziel hin auszugehen, an dem sich das Zusammenspiel von Konfiguration und Handlung orientiert. Bei einem Text wie Gravity's Rainbow, der literarhistorisch einschlägig vor allem aufgrund seines großangelegten Experiments mit den Formen der Darstellung ist, wäre es jedoch gleich aus zweierlei Gründen irreführend, das Ausgesagte gegen Aussage und Aussageweise ausspielen zu wollen: Rückte man allein die choses in den Vordergrund, ließe sich der Text als shistorischer Roman` (miss-)verstehen und gattungstheoretisch sortieren. Konzentrierte man sich umgekehrt bloß auf die mots, erschiene er als avantgardistisch und ließe sich wahlweise als l'art pour l'art abtun oder als sunreadable in die Ecke werfen. Pynchons Prosa jedoch zeichnet sich dadurch aus, dass sie ihre Erkenntnisobjekte mit immer neuen Formen der Darstellung korreliert, die sie an den jeweiligen Gegenständen eigens entwickelt (vgl. dazu auch Lachmann 2020). Zwar weist sie narrative Züge auf. Doch statt einer syntagmatischen Gerichtetheit ihrer Narrateme inszeniert sie paradigmatische Verknüpfungen, die auf dem Prinzip der Similarität beruhen, einander substituieren und dabei doch auf die Phantasmen der Texte bezogen bleiben. Diese zielen also weniger auf spannungsgeladene Erzählungen, als dass sie komplexe historische Konstellationen umkreisen. Der eindeutigste Hinweis darauf, dass es Pynchon in Gravity's Rainbow um etwas anderes als das Erzählen einer Geschichte geht, besteht denn auch darin, dass er auf das epische Präteritum verzichtet und für die Darstellung konsequent das Präsens wählt.

Die erzählte Welt, das >Universum` von Gravity’s Rainbow, umfasst weite Teile der >westlichen 'Welt, vor allem Großbritanniens und Kontinentaleuropas, Norddeutschlands sowie der USA, mit historisch diskontinuierlichen Nebenschauplätzen in den Niederlanden, Deutsch-Südwestafrika oder auf Mauritius, 
und lässt sich als komplexe Ereigniskette im Sinne eines massendynamisch fundierten Geschichtsprozesses begreifen, der sich auf den Zeitraum zwischen dem 18. Dezember 1944 und dem 14. September 1945 konzentriert. Schnell wird ersichtlich, dass die raumzeitliche Ordnung der Diegese, und damit die histoire, in der Erzählweise, und hier vor allem im récit, aufgehoben wird. Dadurch entsteht der Effekt einer gewissen >Außerzeitlichkeit` oder halluzinatorischen Simultaneität der Ereignisse, in der die unterschiedlichen Ebenen der historischen Zeit verschwimmen, so dass Vergangenes und Gegenwärtiges sich vermischen und zeitgleich erscheinen. Dabei reichen zentrale Analepsen zurück bis zum Genozid an den Herero nach General von Trothas Vernichtungsbefehl des Jahres 1904, bis zur Ausrottung der Dodo-Vögel auf Mauritius um 1681 oder bis zur Kolonialisierung Nordamerikas durch britische Puritaner wie John Winthrop, der die Massachusetts Bay Colony mit seiner Flotte aus elf Schiffen und siebenhundert Siedlern im April 1630 erreichte. Prolepsen hingegen weisen historisch voraus auf die Zeit der Textgenese zwischen 1963 und 1973, zugleich - als comicartige Science Fiction aber auch weit darüber hinaus, da Konstrukte wie die utopisch-dystopische Raketenstadt, die zunehmend Gestalt annimmt, auch heute noch Zukunftsmusik sind. Dominiert wird die erzählte Welt dagegen von der Darstellung der »Zone« (Pynchon 1981, 439-964) als »Interregnum« (Pynchon 1981, 461), einem Raum außerhalb der Zeit, in dem alle Regeln, Gesetzmäßigkeiten, Ordnungen und Systeme des menschlichen Zusammenlebens aufgehoben sind. In dieser singulären Situation grassiert die Anarchie. So beschreibt der Text die Zeit nach dem Victory in Europe Day vor allem als »ein großes, grenzenloses Strömen«, in dem sich die »Völkerscharen [...] über die Schutthalde einer Ordnung [schleppen], einer europäischen und bürgerlichen Ordnung, von der sie noch nicht wissen, daß sie zerstört ist für immer« (Pynchon 1981, 857-859). Allerorts, teils verteilt um reale Örtlichkeiten wie die Mittelwerke in Nordhausen und das dortige Konzentrationslager Mittelbau-Dora, die Heeresversuchsanstalt in Peenemünde-Ost oder die Erprobungsstelle der Luftwaffe in Peenemünde-West, teils irgendwo in der deutschen Provinz, entstehen unwirtliche Enklaven, Schwarzmärkte bilden sich aus, bringen Heterotopien wie Putzis Etablissement hervor, während sich das Schwarzkommando in seiner weit verzweigten Erdschweinhöhle verbirgt. An allen diesen Orten spielen sich kleine Ereignisse und Szenen ab. Am ehesten erfasst das Momentum dieses Ausnahmezustands wohl die angehende Hexe Geli Tripping, die einst für das Emblem des Aggregat 4 Modell gestanden hat und die nicht zuletzt aufgrund ihrer deterritorialisierenden Erfahrung der alljährlichen Walpurgisnacht auf dem Brocken an Tyrone Slothrop gewandt sagt: ">Vergiß jetzt Grenzen. Vergiß Unterteilungen. Es gibt keine.८ [...] >Hier ist alles aufgehoben.« " Denn aus ihrer Sicht ist die Zone ein glatter Raum: "»Du brauchst nur darin mitzuschwimmen.» (Pynchon 1981, 462). 
Die damit angesprochene Konfiguration und die Handlung sind zwei zentrale Teilsysteme der Prosa. Gravity's Rainbow allerdings ist berüchtigt für die rund vierhundert literarischen Figuren, die im Text zueinander in Beziehung stehen und das überschaubare Maß einer üblichen Konfiguration bei weitem überschreiten. Wie in vielen anderen Subsystemen des Textes auch, lässt sich in dieser beispiellosen Expansion der Konfiguration der Gestus einer entschiedenen Überbietung aller bisher dagewesenen literarischen Gepflogenheiten erkennen. Sowohl im Hinblick auf die Gesamtheit der Figuren als auch auf ihre >Individualität bleibt dieser Schritt nicht folgenlos: Während die schiere Anzahl an Figuren allein schon für eine gewisse Orientierungslosigkeit sorgt, weil diese leicht verwechselt werden können und in der Masse wenn nicht untergehen, so doch zumindest verschwimmen, erscheint die daraus resultierende Konturlosigkeit als Effekt durchaus adäquat, um das `Kriegsgeschehen im weiteren Sinne als eine nie gekannte Massendynamik zu literarisieren. Die Vielfalt und Heterogenität der Konfiguration lassen sich daher bestenfalls andeuten. Ohne damit etwaige Ordnungsprinzipien postulieren $\mathrm{zu}$ wollen, lassen sich doch zumindest einige Beobachtungen am Text festhalten. So fällt auf, dass einer quantitativ geringeren Zahl von Figuren mit srealen Vorbildern - wie Constance Babington-Smith, Wernher von Braun, John Dillinger, JFK, Fritz Lang, Malcolm X, Iwan Petrowitsch Pawlow, Walter Rathenau, Albert Speer oder der Riege namhafter Politiker von Churchill über Roosevelt und Truman bis hin zu Stalin - das Gros der eindeutig fiktiven Figuren gegenübersteht. Dazwischen finden sich Figuren, die nur leicht fiktionalisiert sind; Major Duane Marvy etwa, der sich vergleichsweise leicht mit dem Chief of the Jet Propulsion Section of the Research and Intelligence Branch of the U.S. Army Ordnance Corps, Major Robert B. Staver, in Verbindung bringen lässt, oder der Oberbefehlshaber der deutschen Raketensektion, Major Weißmann, »ein brandneuer Typ von Militär, halb Geschäftsmann und halb Wissenschaftler« (Pynchon 1981, 626), für den Pynchon die historischen Figuren des Wehrmachtsoffiziers Generalmajor Dr. Walter Dornberger und des SS-Obergruppenführers und Generals der Waffen-SS Dr. Hans Kammler adaptiert. Ähnlich sieht es mit den kollektiven Figuren aus. Hier gibt es eine Reihe bekannter Konzerne - AEG, Agfa, American Food \& Drug, Imperial Chemical Industries, I.G. Farben, General Electric, Krupp, Shell, Siemens, Stinnes, Telefunken, Thyssen, Volkswagen usw. -, die von fiktiven Korporationen, wie der Psychochemie AG, der Spottbilligfilm AG oder der Slothrop Paper Mill ergänzt werden.

Den Figuren Pynchons wird nachgesagt, dass sie teils wenig komplex, ızweidimensional oder, in den Worten Elfriede Jelineks, schlicht »objekte einer überdimensionierten comic-book-welt« seien (Jelinek 1976, 39). Es mag etwas daran sein. Allerdings ist die in dieser Einschätzung mitschwingende, abwertende Konnotation der >Oberflächlichkeit` nur dann bedeutend, wenn man davon ausgeht, literarische Figuren müssten auch in der Postmoderne noch den Stoff für Charakter-Interakti- 
ons-Dramen hergeben. Diesen schon im achtzehnten Jahrhundert einsetzenden Trend zur Psychologisierung literarischer Figuren unterläuft Pynchon, indem er sie auf das reduziert, was sie als fiktive anthropomorphe Individuen ausmacht: ihre Funktion als isotopiekonstitutive Aktanten innerhalb der Matrix einer Konfiguration, in der sie sich durch distinktive Merkmale von den übrigen Figuren unterscheiden und sindividualisieren`, ansonsten aber Vehikel bleiben, um bestimmte konfigurationskonstitutive Oppositionen, diskursive Positionen oder Ideen zu transportieren. An den wiederkehrenden Streitgesprächen zwischen Edward W.A. Pointsman, F.R.C.S., und Roger Mexico, die beide für die britische Political Warfare Executive arbeiten, lässt sich das gut veranschaulichen, stehen sich hier doch ein Behaviorist und ein Statistiker mit ihren jeweiligen Habitus als Kontrahenten gegenüber. Dementsprechend erkennt Pointsman in Mexico nur den »jungen Anarchisten in seinem roten Schal« (Pynchon 1981, 145), dem er mit Pawlows Ideal einer mechanistischen Erklärung der Welt kommt: ")Seine Zuversicht gründete auf dem festen Glauben an eine rein physiologische Grundlage des psychischen Lebens. Keine Wirkung ohne Ursache, und eine klare Kette von Verbindungsgliedern. « Mexico, der aus Pointsmans Sicht die gesamte, verderbte Generation der jungen Wissenschaft verkörpert, reagiert höflich, aber bestimmt, indem er darlegt, dass Denkmodelle wie »Ursache-und-Wirkung « aus Sicht der modernen Statistik als Erklärung für den Weltenlauf längst überholt sind: "»Der nächste große Durchbruch kommt vielleicht erst dann, wenn wir den Mut haben, Ursache-undWirkung radikal zum Abfall zu schmeißen und in einer ganz anderen Richtung neu anzufangen.« (Pynchon 1981, 145). Dem Rätsel der exakten Übereinstimmung zwischen den mit Frauennamen versehenen Sternchen, mit denen der US-amerikanische Lieutenant Tyrone Slothrop die Orte seiner sexuellen Abenteuer auf einem Stadtplan Londons markiert, und jenen Stellen, an denen nur geringfügig zeitversetzt die deutschen V2-Raketen niedergehen, versucht er daher mit dem stochastischen Mittel der univariaten diskreten Wahrscheinlichkeitsverteilung nach Siméon Denis Poisson auf die Spur zu kommen. Indem die Figuren solche diskursiven Positionen verkörpern, formiert sich die Konfiguration als ein Stück figurativer Diskursgeschichte, die Pynchon im Sinne der Polyphonie orchestriert. Ob diese Figuren weniger komplex sind als klassische Charaktere, sei einmal dahingestellt. Aber wahrscheinlich ist die Provokation, dass hierbei mit dem Subjektstatus der einzelnen Figuren zugleich auch das aufklärerische Postulat von der Autonomie des modernen Individuums kassiert wird, einfach zu groß.

Mit Blick auf die Handlung ist zu konstatieren, dass man eine komplexitätsreduzierende Synopsis von Gravity's Rainbow vornehmen würde, wenn man den zentralen Erzählfokus auf den amerikanischen Lt. Tyrone Slothrop legte, der in London für ACHTUNG arbeitet und auf die vom europäischen Festland abgeschossenen, deutschen V2-Raketen mit Erektionen reagiert, sodass er sich 
auf die Suche nach der mysteriösen Geheimwaffe macht. Denn tatsächlich wartet der Text mit einer derartigen Vielzahl von Handlungselementen auf den unterschiedlichen historischen Ebenen der Zeit auf, dass solch eine Zusammenfassung - allein gemessen an der radikal expandierten Konfiguration - zwangsläufig als insuffizient erscheinen muss. Mehr noch zeugen `Inhaltsangaben wie diese doch vom letztlich vergeblichen Versuch, elaborierte Prosa in das Prokrustesbett der Gattungstheorie zu zwängen und einen Text wie Gravity's Rainbow zu behandeln wie einen $\mathrm{x}$-beliebigen, vielleicht etwas sabgefahrenen`, WK-Zwo-Roman oder eine mittelalterliche Queste im technohistorischen Gewand des London Blitz. In Gravity's Rainbow laufen die unzähligen Fäden der Handlung aber nicht schematisch zusammen, sondern auseinander.

Die im Zusammenhang mit dem Zerfall der Diegese in kleine und kleinste Räume - oftmals übrigens Schlafzimmer oder andere klandestine Orte - sowie Ereignisse zu beobachtende Tendenz zu Auflösung und Zerstreuung lässt sich im Bereich der Handlung wiedererkennen und mit Jean-François Lyotard als mit La condition postmoderne (Lyotard 1979) assoziierte Kritik an der Macht der großen Erzählungen und als Fragmentarisierung übergeordneter Zusammenhänge begreifen. In handlungstheoretischer Sicht bleibt der Text dabei paradox. Denn im Grunde ereignet sich nicht allzu viel - agonistische Handlungssequenzen jedenfalls sucht man vergeblich - und doch kann nicht davon die Rede sein, Gravity's Rainbow sei handlungsarm. Schließlich weist der Text vielfältigste Handlungselemente auf, die quer durch das übliche, gattungsspezifische Spektrum führen und die allesamt unterhaltend sind. Es gibt also immer wieder kleine Handlungselemente, denen die Funktion zukommt, die Entwicklung des Textes zu garantieren. Bloß lassen sich diese nicht ohne weiteres in eine übergeordnete, ssinnhafte Struktur integrieren. Sie sind in erster Linie funktional.

Greift man exemplarisch eine der vielleicht spektakulärsten Handlungssequenzen heraus, die wilde Verfolgungsjagd durch die Mittelwerke, die dank der Hilfe eines dubiosen deutschen Wissenschaftlers vom Institut für Mathematik der TH Darmstadt sprichwörtlich `glimpflich` verläuft (Pynchon 1981, 484), weil es Slothrop einmal mehr gelingt, sich dem Zugriff von Marvys Müttern zu entziehen, dann stellt sich nach der Lektüre doch die Frage, worin die narratologische Funktion dieser Passage bestanden haben mag, wenn nicht darin, en passant Architektur und Organisationsstruktur der Mittelwerke vermittelt zu haben. Damit aber liefert Pynchon einmal mehr gründlich recherchierte Informationen über einen unlängst größeren, wenn auch weiterhin im Verborgenen bleibenden historischen Zusammenhang. Als Form der Darstellung jedoch wählt er - im Bruch mit allen Konventionen - das Genre des Slapsticks, erinnert Slothrops Flucht auf dem mit Sprengkopf-Segmenten beladenen Miniaturzug doch nur zu sehr an entsprechende Szenen aus Stummfilmklassikern wie Larry Semons The Show (1922) oder 
Monty Banks' Chasing Choo Choos (1927). Wenn sich die Figur dabei zu tarnen versucht - »Slothrop klettert in den Overall, kämmt sich seine Haartolle tief in die Stirn, zieht sein Taschenmesser heraus und säbelt sich an beiden Seiten ein Stück Schnurrbart ab« -, dann ist die naheliegende Parodie dabei inklusive. Professor Glimpf: »)Jetzt sehen Sie aus wie Hitler. Jetzt wird man Sie erst recht umbringen wollen.« Und der Erzähler kommentiert: »Deutscher Humor« (Pynchon 1981, 484).

Zusammengenommen hat das eine entscheidende Konsequenz: Die Einsicht, dass es sich bei Gravity's Rainbow beim besten Willen nicht um einen Roman handeln kann. Folgerichtig wird er im amerikanischen Original auch nicht als ein solcher ausgewiesen. Denn:

In den Prosatexten überwuchert eine wilde Semiose die gegebenenfalls vorhandenen romanaffinen Ordnungsmomente wie erzählerischen Fortgang, Charakterentwurf von Figuren, Stabilität einer vorgestellten Welt etc. Man kann folglich die Prosa als eine Reflexion des Romans bestimmen, nämlich als Schreibweise, die den Roman radikalisiert, seine Formmomente durch textuelle Überwucherungen aushebelt und einen amplificatio-Modus etabliert, der die Textarbeit richtungslos oder besser: plurifokal werden lässt.

(Simon 2018, 417)

Treffender lässt es sich kaum sagen. Nur auf das Subsystem der Handlung bezogen, bliebe zu ergänzen, dass Pynchon verschiedene Verfahren nutzt, um das ordnungsstiftende Moment des gewöhnlich als Verkettung von interpersonalen Interaktionen vorgestellten Handlungsverlaufs zu irritieren: Dazu zählt die Anachronie im narratologischen Bereich der Ordnung genauso wie das Zusammenspiel von zeitdeckenden szenischen Darstellungen, Dehnungen und Pausen des Verhältnisses zwischen Erzählzeit und erzählter Zeit durch umfangreiche Schilderungen von Subjektsituationen, imaginären Evokationen oder Deskriptionen mit Realitätseffekt, die im Bereich der Dauer mit extremen Raffungen bis hin zu die Schnitt- und Montagetechnik des Films imitierenden Ellipsen kombiniert werden. Entscheidender aber noch erscheint der Umstand, dass Pynchon die Nutzung der üblichen Formen zur Motivation von Handlung verweigert. Anstelle von Intentionalität betont er die Kontingenz. So scheinen die Figurenhandlungen um Major Weißmann und Tyrone Slothrop nicht etwa auf den übermächtigen Willen eines Nazi-villain, auf schicksalhafte Begegnungen oder befohlene Missionen zurückzugehen, sondern auf ein Tarot (vgl. Pynchon 1981, 1172-1185). Ins Esoterische gewendet, eröffnet der Text alternative Motivationen für die Handlung: zum einen die simple Synchronizität des zeitgleichen oder zeitnahen Auftretens zweier Ereignisse, die zwar in einem motivierten, nicht aber in einem kausalen Zusammenhang stehen, zum anderen die rein assoziative (oder projektive) Verknüpfung von verschiedenen Ereignissen. Beide Varianten stellen das aufgeklärte westliche Denken radikal in Frage. Statt der zu erwartenden Kausalität der Handlungsführung nach 
dem Prinzip von Ursache und Wirkung, bekanntermaßen Gegenstand bereits des Streits zwischen Pointsman und Mexico, herrscht die reine Kontingenz vor und, als Reaktion darauf, grassiert die Paranoia. So offenbart Slothrop seinem einzigen Freund, dem britischen Lieutenant Oliver »Tantivy« Mucker-Maffick, seinen Verfolgungswahn:

Er ist jetzt besessen von der Idee einer Rakete, auf der sein Name geschrieben steht. Wenn sie ihn tatsächlich erwischen wollen (und »SIE« umfaßt Möglichkeiten, weit, weit über Nazideutschland hinaus), dann ist das der sicherste Weg, es kostet sie ja schließlich nicht die Welt, seinen Namen auf jede einzelne ihrer Raketen draufzupinseln, oder?

(Pynchon 1981, 43f.)

Die Thematisierung des mechanistischen Geschichtskonzepts, dessen unterstellte Kausalität durch die V2-Rakete in jenem Maß erschüttert wird, in dem sich bei ihr Wirkung, Explosion, und Ursache, das heulende Signal des nahenden Geschosses, umkehren, schreibt sich über die rhetorische Figur des hysteron proteron unmittelbar in die Prosa ein. So lebt Captain Geoffrey »Pirat« Prentice im Bewusstsein: »Er wird das Ding nicht hereinkommen hören. Es bewegt sich schneller als der Schall. Die erste Nachricht, die man erhält, ist die Explosion. Danach - wenn's einen dann noch gibt -, danach erst, hört man das Geräusch ankommen « (Pynchon 1981, 16). Diese Zeitenwende reflektiert der Text philosophisch, wenn er die rhetorische Frage stellt, ob »die Nachkriegszeit nur noch aus Zufallsereignissen bestehen [wird], isoliert, von einem Augenblick zum nächsten neu erschaffen? Ohne Verbindungsglieder? Ist dies das Ende der Geschichte?« (Pynchon 1981, 93) und darüber die Diskussion über Postmoderne und Posthistoire aufruft. Er übersetzt die Denkfigur aber auch in die unterschiedlichen Subsysteme der Textur, etwa wenn die Wissenschaftler in der Weißen Visitation dazu übergehen, »den Krieg selbst einmal als Laboratorium « zu betrachten und beginnen, die Erkenntnisse der V-Waffen-Entwicklung auf andere Wissensbereiche zu übertragen: »Wenn die V 2 einschlägt, dann kommt zuerst die Explosion und danach das Geräusch des Fallens ... das heißt, die normale Reihenfolge der Reize läuft umgekehrt ab ... (Pynchon 1981, 82), wie der Neurologe Dr. Kevin Spectro erkennen muss. Und schließlich bleibt auch die Darstellungsweise der Prosa nicht unberührt von dieser neuen Denkmöglichkeit, was sich daran ablesen lässt, dass mit der Chronologie auch die übrigen Ordnungsmomente des Textes aufgelöst werden. So ist es keine Seltenheit, wenn Tschitscherin und sein Fahrer Dschabajew bei einer Scheibe Haschisch, die sie Slothrop entwendet haben, über dem Ergebnisprotokoll einer Natrium-AmytalSitzung brüten und das eigentliche Ereignis der Befragung unter Einfluss des Wahrheitsserums erst danach Thema ist (vgl. Pynchon 1981, 609-612) oder wenn die der Droge Oneirin nachgesagte Eigenschaft zur Zeitmodulation auf der Handlungsebene dahingehend genutzt wird, den unausweichlichen kriegerischen $\mathrm{Zu}$ - 
sammenstoß zwischen dem U-Boot der argentinischen Anarchisten und dem USZerstörer John E. Badass so zu beeinflussen, „daß sich die beiden fatalen Kurse auf dem mürben Meeresecho dieser Nacht zwar räumlich kreuzen, nicht aber in der Zeit. Nicht auch nur annähernd in der Zeit, heh, heh« (Pynchon 1981, 608).

Es sollte in diesem Zusammenhang nicht verschwiegen werden, dass mit dem traditionellen Bild der Geschichte auf einer Metaebene zugleich auch die Funktionsweise des Textes selbst ironisiert wird, wobei sich zeigt, dass der Text insgesamt starke karnevalistische Tendenzen aufweist. So stellt sich Slothrop, der in der Zone eine Zeitlang den Jungen Ludwig begleitet, der sich auf der Suche nach seinem verlorenen Lemming Ursula befindet, die Frage, weshalb er dies alles überhaupt tut. Wie sich hieran zeigt, ist er sich selbst - und damit nicht zuletzt auch seiner eigenen Funktion im Text - nicht sicher. Es kostet ihn einiges an Überlegung, sich darauf zu besinnen, was die Leserschaft von ihm erwartet, bis ihm schließlich einfällt:

Yeah! Yeah, was aus dem Imipolex G wurde, dem ganzen Jamf-Krampf und dem S-Gerät, ein hartgesottenes Privatauge soll ich hier darstellen, im Alleingang gegen den Rest der Welt und gewinnen, meinen Freund rächen, den SIE getötet haben, meine Papiere wiederkriegen und dieses Mystery-Gerät aufspüren.

(Pynchon 1981, 876)

So wie Slothrop Assoziationen zu den Handlungsschemata gängiger Genres der Populärliteratur wie dem Detektivroman, dem Agententhriller, dem Abenteuerroman, der Vendetta oder der >Queste « weckt, werden auch die stereotypen Formen und Formate der Gattungstheorie parodiert. Denn als >professionelle Figur ist sich Slothrop bewusst, wie unrealistisch diese sind, während sie der Leserschaft in der Kombination mit den übrigen Darstellungsformen doch ein intimes Wissen über die Ontologie ihrer Gegenwart vermitteln. Indem es lauthals singend aus ihm herausbricht, bekennt er in schonungsloser Offenheit die Unwahrscheinlichkeit seines plots, den man wohl nirgendwo anders als in der Literatur akzeptieren würde: »in Wahrheit isses GANZ WIE - / NACH NER STECKNADEL IN EIM HEUUUSCHOBER SUCHEN!« (Pynchon 1981, 876). Auf der Metaebene reflektiert er damit zugleich die Machart des Textes, der ganz gezielt mit Versatzstücken populärer Genres arbeitet.

Praktisch folgt aus der Diagnose des `Endes der Geschichte`, dass die Handlung in unzählige Handlungssplitter zerfällt und daher nicht anders als anekdotisch dargestellt werden kann. Was sich in einem empirischen Praxistest leicht überprüfen lässt - man braucht nur den Versuch zu unternehmen, jemandem zu erzählen, wovon Gravity's Rainbow handelt, schon wird man sich dabei ertappen, wie man die skurrilsten, gewitztesten oder obszönsten Anekdoten zum Besten gibt -, verrät viel über die Machart des Textes: So lässt er sich symbolisch wahlweise als Konglomerat kleinerer Bauteile, als `buntscheckiges` Lumpen- 
gewand oder als eine aus verschiedenfarbigen Fäden generierte, changierende Textur vorstellen. Ein globales Handlungsschema der histoire als abgeschlossene und sinnhafte Struktur, wie die Erzähltheorie es erfordert, lässt sich hingegen nicht plausibilisieren. Es existiert kein erkennbares Erzählziel. Wollte man etwa behaupten, es handele sich um eine Queste, wie in manchen Sekundärtexten zu lesen ist, dann müsste man begründen können, weshalb diese weder zu einem Ziel führt noch einen Protagonisten, geschweige denn einen `Helden`, aufweist. Die vermeintliche Hauptfigur löst sich einfach auf. Und sollte die Figur zwischenzeitlich selbst einmal dem Irrtum aufsitzen, etwas Sinnvolles getan zu haben, indem sie sich auf das europäische Festland versetzen lassen und die Fährte der V 2 aufgenommen hat, dann wird diese Annahme in der narration sogleich als Illusion entlarvt. Es ist »Slothrops tumbes, leeres Herz«, das zu ihm spricht und sagt:

das Schwarzgerät, Freundchen, ist kein Gral - dies ist es nicht, wofür das G in Imipolex G steht. Und du bist kein ritterlicher Held. Das Beste, womit du dich vergleichen kannst, ist Tannhäuser, der singende Einfaltspinsel - du hast unter einem Berg geweilt in Nordhausen, du singst ganz gerne mal zu deiner Ukulele ein, zwei Lieder, und haste nicht auch das Gefühl, daß du hier in einem schmatzenden Sündenpfuhl rumlatscht, Slothrop? [...] aber was du getan hast, war, eine fremde Reise anzutreten, von irgendeiner Frau Holda oder Venus in irgendeinem Berg, und ein fremdes Spiel mitzuspielen ... von dem du weißt, daß es in seinem nicht wegzuleugnenden Kern ein böses Spiel ist. Du spielst mit, weil du nichts Besseres zu tun hast, aber wird es davon weniger verwerflich? Und wo ist der Papst, dessen Stab grünen wird für dich?

(Pynchon 1981, 570)

Die hierin zum Ausdruck gebrachte ironische Selbstreflexivität des Textes nimmt zunehmend die ätzenden Züge der Satire an. Und die lanx satura verleibt sich die zeitgenössische Form des amerikanischen Vaudeville ein (Dimeglio 1973), nach dessen anarchischem Prinzip sie den Text organisiert; ganz ohne in sich geschlossene Handlung, lediglich lose verknüpft nach dem Prinzip der Nummernrevue. Von dort aus erklärt sich auch die gewählte Form der Darstellung; eine Darstellung, die Assoziationen zum einfachen, teils derben Volkstheater, zur Schaubudenkunst und zur Burleske weckt. Traditionell treten im Vaudeville (Mattes 1983), dieser Urform des Trink- und Spottlieds sowie der Urszene des Slapstick, Kleinkünstler auf: Musiker, Komödianten, Bauchredner, Magier, Illusionisten, Dresseure, Akrobaten und Gymnasten; was allein schon all jene Figuren mit militärischem Rang und scheinbar wichtigen Funktionen der Lächerlichkeit preisgibt, die der Text aufbietet. Darüber hinaus liefert das Vaudeville-Format eine mögliche Tradition für die in den Text montierten Songs und Couplets, jene mehrstrophigen witzig-zweideutigen politischen oder satirischen Lieder mit eingängigen Refrains, in die dieser unversehens immer wieder abdriftet. So findet sich Slothrop, als er in einen weißen Zoot-Anzug gekleidet in die Zürcher Spitzel- und Informantenszene eintaucht, urplötzlich in einer ganz anderen Szene wieder, nämlich einer chorus line, die 
»nicht, wie üblich, aufgeteilt in Jungen und Mädchen, sondern in Wärter und Irre« (Pynchon 1981, 410) ist, deren Auftritt in einem Nebentext detailliert beschrieben wird und die Slothrop zwischen den Strophen Informationen über die unterschiedlichsten geheimen Erfindungen oder »die sexuellen Gewohnheiten von hundert ausgewählten Aufsichtsräten, Fabrikgrundrisse, Codebücher, Verbindungen und Zahltage« (Pynchon 1981, 410) offerieren. Sie sind die

\section{LOONIES ON LEAVE!}

Here we come foax - ready or not!

Put your mask on, and plot your plot,

We're just laughin' and droolin' all-over

the sleigh,

Like a buncha happy midgets on holiday!

Oh, we're the LOONIES ON LEAVE, and

We haven't a care -

Our brains at the cleaners, our souls at the Fair, Just freaks on a fur-lough, away from the blues, As daffy and sharp as - the taps on your shoes!

Hey, we're passin' the hat for - your frowns and your tears,

And the fears you thought'd never go 'way -

Oh take it from a loony, life's so dear and swoony,

So just hug it and kiss it to-day!

La-da-da, ya-ta ya-tata-ta \&c ...

(Pynchon 1973, 259)

\section{IRREN AUF URLAUB!}

Kuckuck, Leute, grüß euch Gott, Maske auf und ab Komplott, Laßt uns sabbern, tanzen, lachen, Fröhliche Zwerge, die Ferien machen!

Ja, wir sind die IRREN AUF URLAUB, Sind weich auf der Birne, die Seelen Beim Feiern, beim Waschen die Hirne, Verrückte im Freigang, der Anstalt entflohn, Nicht für dumm zu verkaufen Wir sind es ja schon! Und geht der Hut rum, so schmeißt uns was ein Eure Sorgen und Tränen - wir steppen sie klein. Denn glaubt uns Idioten: das Leben ist schön! 
Laßt euch nicht verschrecken,

Dann wird es schon gehen!

La-da-da, ya-ta ya-ta ta-ta \&c

(Pynchon 1981, 408f.)

Die Änderung des Namens des Theaters, in das die V2 des Textbeginns einschlägt, von »Orpheum « zu »Orpheus« verweist im Übrigen auf das Verbot des Blicks zurück. In einem nach dem thrakischen Sänger Orpheus benannten Vergnügungstempel, der - wie wir aus dem Text erfahren - auch die Leserschaft von Gravity's Rainbow beherbergt, die vor Ort den Mindless Pleasures frönt (so Pynchons ursprüngliche Titelidee für den Text), könnte die satirisch geschärfte Kritik am verlorenen Geschichtsbewusstsein der Gesellschaft des Spektakels (Debord 1996) nicht drastischer ausfallen. Auf der Metaebene handelt es sich bei Gravity's Rainbow also um einen Text, der gegen die Geschichtsvergessenheit der Gegenwartskultur angeht, indem er historische Ereignisse in das Gewand populärer kleiner Formen kleidet und sich immer wieder dem ungehemmten Fluss des höheren (wie niederen) literarischen Schabernacks überlässt. Gegen Kontinuität und Linearität wendet sich Gravity's Rainbow überdies auch im literarischen Verfahren des Witzes, der sich - in überraschender struktureller Verwandtschaft zum Tarot - auf Ereignisse bezieht, die Lessing zufolge, "weiter nichts miteinander gemein haben, als daß sie zugleich geschehen«. Lessing stützt sich auf die Symbolik des Fadens und der Textur, wenn er ausführt, dass die Kunst des Witzes darin besteht, die heterogenen Begebenheiten so miteinander zu verbinden, »ihre Faden so durch einander zu flechten und zu verwirren, daß wir jeden Augenblick den einen unter dem andern verlieren, aus einer Befremdung in die andere gestürzt werden [...]«. Die Struktur, die aus »der beständigen Durchkreuzung solcher Fäden von ganz verschiednen Farben« hervorgeht, die man im Sinne der strukturalen Semantik als Isotopien bezeichnen könnte, beschreibt Lessing als »eine Kontextur, die in der Kunst eben das ist, was die Weberei Changeant nennet: ein Stoff, von dem man nicht sagen kann, ob er blau oder rot, grün oder gelb ist; der beides ist, der von dieser Seite so, von der andern anders erscheinet; ein Spielwerk der Mode, ein Gaukelputz für Kinder« (Lessing 1973, 368f.). Es ist eines der markantesten Merkmale von Gravity's Rainbow wie von Pynchons Prosaprojekt überhaupt, dass das Verfahren des Witzes, von der einfachen Verfremdung bis hin zu den komplexen Interdiskursivitäten, alle Dimensionen des discours erfasst. Das beginnt auf der elementarsten Ebene mit einfachen Kalauern, etwa über das ornithologische Schillern der Farben, die in der Flamme der V2-Rakete oszillieren, wenn diese beginnt, sich von der Bodenplatte zu erheben, was dazu führt, dass das Geschoss in SS-Kreisen »Pfau Zwei« genannt wird (Pynchon 1981, 354), reicht über Gags wie den Namen des Kurorts »Bad Karma«, die auf Grundlage von Bilingualität generiert werden (Pynchon 1981, 715), bis hin zu albernen, immerhin reinen Reimen wie 
jenem »Hallo Kamerad, brauchst'n Opiat?« mit dem Albert Krypton, seines Zeichens Lazaretthelfer an Bord der USS John E. Badass, versucht, seinen Stoff an den Mann zu bringen (Pynchon 1981, 929). Etwas komplexer sind demgegenüber die Raketen-Limericks, die Marvys Mütter zu einer in amerikanischen Studentenverbindungen bekannten Melodie zu schmettern pflegen, zum Beispiel:

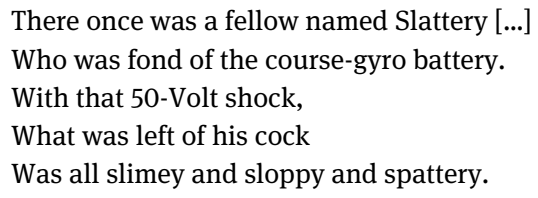

Diese Limericks reichen aber nicht an die Couplets heran, in denen mitunter auch ernsthafte Töne angeschlagen werden. So gibt es unter den Soldaten des Schwarzkommandos und ihren Familien mit der Fraktion der Leeren auch eine Gruppe, die den versuchten Völkermord an ihrer Gemeinschaft in den Wunsch nach kollektivem Selbstmord ummünzt. »[E]uropäisiert in Sprache und Denken« und »abgeschnitten von der alten Einheit ihres Stammes" geben sich die Otukungurua der Dekadenz ihrer Kolonisatoren hin und träumen vom Tag, »an dem der letzte Zonen-Herero tot sein wird«, dem »endgültigen Nullpunkt einer voll ausgelebten kollektiven Geschichte« (Pynchon 1981, 498). Allerdings stehen sie damit in Konflikt zum übrigen Schwarzkommando, weshalb ihr Anführer Josef Ombindi versucht, Enzian auf seine Seite zu ziehen. Dazu macht er ihn

\section{SOLD ON SUICIDE}

Well I don't care-for, th' things I eat, Can't stand that boogie-woogie beat -

But I'm sold, on, suicide!

You can keep Der Bingle too, aAnd that darn »bu-bu-bu-boo, « Cause I'm sold on suicide! 
But I'm sold, on, suicide!

Don't like either, the Cards or Browns, Piss on the country and piss on the town, But I'm S.O.S.

(Pynchon 1973, 320)

\section{SCHARF AUF SELBSTMORD}

Tcha, was ich esse, schmeckt mir schal, Der Boogie-Woogie ist mir ganz egal, Denn ich bin scharf, auf, Selbstmord!

Auch vor Bing Crosby laßt mir meine Ruh, Vergeßt sein blödes »bu-bu-bu-boo«, Ich bin nur scharf auf Selbstmord!

Die Lebensmittelmarken könnt ihr sparen, Und auch die Muttis, die mal Baby-Vamps waren, Denn ich bin scharf, auf, Selbstmord!

Baseball hab ich genauso satt, Scheiß auf das Land, scheiß auf die Stadt, Denn ich steh nur auf Selbstmord [...].

(Pynchon 1981, 501)

Und der Erzähler, der sich auch sonst nicht eben zurückhält, einen sich anbahnenden Flirt zwischen Katje Borgesius und Enzian mit Sätzen wie »Ah-ha. Das ist der Stoff, für den ihr hier seid, Freunde! « (Pynchon 1981, 1035) kommentiert, oder seine Leserschaft aufgrund ihrer Naivität mit Aussagen wie »Ihr werdet Ursache-und-Wirkung wollen« foppt (Pynchon 1981, 1037), lässt uns wissen, dass dieser Song »eine ziemlich summarische Zurückweisung der Dinge des Lebens« beinhaltet (Pynchon 1981, 501). Zum Ende des Textes, als auch dieser beginnt, sich zunehmend in kleinere, mit individuellen Überschriften versehene Fragmente aufzulösen, darunter »MOM SLOTHROPS BRIEF AN BOTSCHAFTER KENNEDY« (Pynchon 1981, 1068f.), finden sich zudem einzelne Kapitel, die sich mit dem Sprachwitz auseinandersetzen. In einem dieser Kapitel, das unter anderem die »KIFFER-KADENZ« enthält (Pynchon 1981, 1073f.), streiten Säure Bummer, Slothrop und Seaman Bodine über »DIE WENDUNG >ASS BACKWARDS« (Pynchon 1981, 1069-1076), während das Kapitel »SHIT 'N' SHINOLA« eine ausführliche Exegese dieser auf einem Isotopiebruch basierenden und insofern als Witz zu bezeichnenden Redensart enthält, die dabei immer weiter variiert wird, bevor das Kapitel »EIN ZWISCHENFALL IM TRANSVESTITENKLO« einmontiert wird und so weiter und so fort (Pynchon 1981, 1076-1078). 
Nicht unerwähnt sollte bleiben, dass Konfiguration und Handlung beileibe nicht die einzigen generativen Subsysteme sind, die von Pynchon radikal überstrapaziert werden. Es sind nur wenige andere Autoren bekannt, die das Konzept der Dialogizität ähnlich ausreizen. An den frühen Texten kritisiert Pynchon seinen Umgang mit Dialogen noch als einen »Fall von Schlechtem Ohr« (Pynchon 1985, 10). Wie Gravity's Rainbow eindrucksvoll zeigt, hat er das Zuhören zwischenzeitlich sehr gut gelernt. Sein Ohr steht längst nicht mehr nur jenen amerikanischen Stimmen offen. Vielmehr mischt er britisches und amerikanisches Englisch mit Deutsch, Französisch, Hebräisch, Herero, Japanisch, Kasachisch, Niederländisch, Russisch und Spanisch, flicht Straßenslang, Songtexte, Filmdialoge und Comic-Sprechblasen ein, nutzt minoritäre Ethno-, Sozio- und Regiolekte, pflegt ein Faible für Rotwelsch, genauso wie er volkstümliche Sagen und Legenden, Kinderreime und die professionelle Sprache von Militär und Wissenschaft ins Spiel bringt. Neben der auf diese Weise entstehenden Polyphonie ist es die exzessive Interdiskursivität des Texts, die über seine Intertextualität noch hinausgeht und als sein wohl charakteristischstes Merkmal gelten kann. Auch dieser Punkt ist literaturtheoretisch einschlägig. Wenn sich das Wissensspektrum moderner Gesellschaften nach C.P. Snow in zwei, nach Wolf Lepenies in drei und jüngsten Auffassungen zufolge in vier und mehr Kulturen differenziert (Pethes 2003, 194-199), die sich untereinander immer weniger $\mathrm{zu}$ sagen haben, so zeichnet sich Pynchons Prosa umgekehrt gerade dadurch aus, dass es ihr gelingt, das sektoriell weit verstreute Wissen literarisch zu integrieren und subjektiv erfahrbar zu machen; selbst wenn es dabei um so entfernte Wissensbereiche wie Physik, Chemie und Elektrotechnik auf der einen Seite und bestenfalls pseudowissenschaftliche wie Astrologie, Parapsychologie und Telekinese auf der anderen gehen sollte; um von Hexerei und anderem ganz zu schweigen. Man kann es nicht anders als virtuos bezeichnen, wie diese Wissensbestände in die Textur eingewebt werden. Teils in die Handlung integriert, fungieren sie teils auch als Aufwertung von Deskription und imaginärer Evokation. Wenn etwa im Rahmen einer Idylle geschildert wird, wie Slothrop im Spätsommer der Zone nach Cuxhaven weiterdriftet - denn er lässt sich treiben, ist ganz passiv, ein Wimpernschlag im Spiel der Zeit -, dann wird die über Sememe wie >Wiesen`, `Regen`, `Schilf` und `Schafe` konstituierte Isotopie der Natur erst behutsam in Richtung Architektur moduliert, bevor sie von einer Beschreibung der "Treppengiebel, die die Fassaden von so vielen dieser alten norddeutschen Häuser krönen«, beinahe unmerklich in die Gefilde der Mathematik übergeht:

Sie halten ihre Form, sie überdauern: Monumente der Analysis. Vor dreihundert Jahren lernten die Mathematiker, den Aufstieg und Fall der Kanonenkugel in Treppenstufen aus Weite und Höhe zu unterteilen, $\Delta \mathrm{x}$ und $\Delta \mathrm{y}$, Stufen, die sie kleiner und kleiner werden 
ließen, immer dichter gegen die Null, während Heerscharen von ewig schrumpfenden Zwergen über die Treppen hinauf- und hinuntergaloppierten und das Getrappel ihrer immer winzigeren Füße gleichmäßiger wurde, sanfter, und endlich ein kontinuierlicher Klang. Unverändert ist dieses analytische Vermächtnis durch die Zeit gereicht worden bis nach Peenemünde, wo es den Technikern die Askania-Filme der Raketenflüge bescherte, die sie anglotzten, Kader um Kader, $\Delta \mathrm{x}$ um $\Delta \mathrm{y}$, ohne davon das Fliegen selbst zu lernen ... Film und Kalkül, Pornographien des Fluges beide ...

(Pynchon 1981, 885)

Wie die phantastischen Gestalten der Zwerge hier die Infinitesimalrechnung bevölkern und unter der Parabel über die Stufen einer Differentialgleichung trippeln, bevor die Deskription wie in einer Kamerafahrt langsam weiterschwenkt, die Giebel als »Mahnungen an Impotenz und Abstraktion« ausblendend und stattdessen eine Gruppe Kinder fokussierend, die im Spiel »von Himmel zu Hölle zu Himmel« (Pynchon 1981, 886) springen, ist schon aller Ehren wert. Es wäre zwar verfehlt, Pynchon zum sschreibenden Ingenieur` zu stilisieren, den manche in ihm sehen, es ist aber durchaus richtig, festzuhalten, dass auch die Sprache der Mathematik ihren Anteil an der komplexen Interdiskursivität von Gravity's Rainbow hat. Der Text selbst nimmt gegenüber dieser Sprache, die er zitiert und die er spricht, eine durchaus kritische Haltung ein. Schließlich vermögen mathematische Formeln als Signifikanten viele, die dieser Sprache nicht mächtig sind, über ihre tatsächlichen Signifikate hinwegzutäuschen:

So wurde der furchtbare Pfad der Rakete buchstäblich reduziert auf bürgerliche Termini, auf Ausdrücke einer Gleichung wie jener eleganten Vermählung von Philosophie und Stahl, von abstraktem Wandel und Drehbolzen aus realem Metall, welche Bewegung unter dem Aspekt der Pendelungsdämpfung beschreibt:

$$
\theta \frac{d^{2} \phi}{d t^{2}}+\delta^{\star} \frac{d \phi}{d t}+\frac{\partial L}{\partial \alpha}\left(s_{1}-s_{2}\right) \alpha=-\frac{\partial R}{\partial \beta} s_{3} \beta,
$$

bewahrend, besitzergreifend - die Kontrolle, die zwischen Scylla und Charybdis ihren Weg zum Brennschluß steuert.

(Pynchon 1981, 378)

Es ist in diesem Rahmen leider nicht möglich, der unbändig wuchernden Interdiskursivität von Gravity’s Rainbow über die Kluft zwischen den ızwei Kulturen hinweg noch weiter nachzugehen. Deshalb sei an dieser Stelle lediglich festgehalten, dass sich analytisch auf dem Weg über die Diskurskombinatorik die der Prosa zugeschriebene `Enzyklopädikı operativer fassen ließe. 


\section{3 theoria: Aspekte einer kynischen Literaturtheorie im Spezialdiskurs}

Die hier nur sehr kursorisch wiedergegebene Analyse beschließend, möchte ich zu guter Letzt noch eine Theoriefigur in den Vordergrund rücken, in der sich der Text im Medium der Literatur selbst reflektiert. Dabei geht es letztlich noch einmal um die Frage, wie die Prosa Pynchons das Verhältnis zwischen der außerhalb von histoire und discours liegenden Lebenswirklichkeit, auf die sie sich mit Hilfe von (para-)realistischen Verfahren bezieht, und ihrer durch histoire und discours realisierten Darstellungsweise ästhetisch reflektiert. Dass sich die dabei entstehende >Friktionalität` als ein Effekt der Maximierung sprachlicher Komplexität erweist, also auf der Poetizität der Prosa beruht, ließ sich hoffentlich andeuten. Doch selbst darüber geht Pynchon noch hinaus: Im Gespräch mit einem Zürcher Schwarzmarkthändler, von dem sich Slothrop Aufschluss über seine frühkindliche Konditionierung durch den Chemiker Laszlo Jamf erhofft, wird er gefragt: »Hinter was bist du her?« Er antwortet: »Äh, Informationen?« (Pynchon 1981, 406), und muss erleben, wie seinem Gegenüber ein »tragikerfüllter Seufzer« entfährt: »Ist es denn ein Wunder, daß die Welt verrückt geworden ist, wenn Information das letzte gültige Tauschobjekt darstellt?« (Pynchon 1981, 407) Tatsächlich ist der Text nicht zuletzt - oder: unter anderem auch - eine groß angelegte Reflexion über die >Wahrheit der technischen Welt‘. Und als Methode für dieses Vorhaben schlägt er eine Strategie vor, die im Text als »operative Paranoia« (Pynchon 1981, 44) bezeichnet wird: »die Überbietung des wahnhaften Kalküls« der seit dem Zweiten Weltkrieg immer »dicht[er] vernetzten Informationssysteme durch die methodische Verrückung, >mit Vernunft zu rasen« (Stingelin 1988, 242). Worum genau geht es also bei diesem rationalen Zeichenwahn? Andernorts im Text wird das Prosaprojekt Pynchons konkretisiert und im Zusammenhang als »Daten-Rückverfolgung « (Pynchon 1981, 909) - im englischsprachigen Original als »data retrieval« (Pynchon 1973, 582) - bezeichnet. Konkret geht es dabei um den Versuch, das im Verborgenen bleibende Netz zwischen staatlichen Organen der in den Krieg involvierten Nationen und privatwirtschaftlichen Konzernen in all seinen Verzweigungen und feinsten Ziselierungen zu rekonstruieren. Der Magnat Lyle Bland scheint darin die Spinne zu sein. Ansonsten erfährt man von dieser Figur nur, dass sie neben einem Posten im Verwaltungsrat der Slothrop Paper Mill - die Familie produziert »Toilettenpapier, Banknoten, Zeitungspapier«, »ein Medium oder Fundament für Scheiße, Geld und das Wort. [...] Scheiße, Geld und das Wort, die drei amerikanischen Wahrheiten, die die Maschine in Gang hielten« (Pynchon 1981, 48) - ein eigenes Institut sowie die Bland-Stiftung betreibt. Internationale Kontakte unterhält er nicht bloß zur I.G. Farben, zu Stinnes, zu Thyssen 
und Krupp, sondern auch zum sonstigen organisierten Verbrechen. Über die vordergründige Interaktionskette der Handlung hinaus geht es dabei auch um die Massendynamik des Geschichtsprozesses. Pynchons Vorhaben gleicht damit dem Versuch einer Ontologie der Gegenwart als Analyse unseres historischen Gewordenseins, wie sie unter dem Vorzeichen einer `Geschichte der Denksysteme auch Michel Foucault betrieben hat. Beiden Projekten gemein ist ihre Kairologie, wie Jürgen Link sie genannt hat, da beide der »Aktualität des historischen Moments (Kairos) mit seinem Ereignis-Charakter zwischen szientifischer, tendentieller Gesetzmäßigkeit und kontingenter Singularität nachspüren« (Link 2018, 19). Ganz ähnlich wie das Projekt Foucaults verknüpft der literarische Text eine archäologische mit einer genealogischen Dimension. Die archäologische Dimension besteht dabei in der »Erschließung und Beschreibung eines Datenraums« (Kittler 2002, 17), der durch die Sedimentierung von Konfiguration und Handlung des Textes in eine Vielzahl von - kontingenten oder intentionalen - Ereignissen erzeugt wird, die sich auf den unterschiedlichen shistorischen und darüber komplexe Konstellationen erzeugen. Die genealogische Dimension hingegen ergibt sich aus der Art und Weise, wie diese unterschiedlichen Ebenen und Elemente im discours miteinander vernetzt werden. Dabei ist die Verknüpfung der historisch zurückliegenden Elemente mit den Phänomenen der Gegenwart von übergeordneter Bedeutung.

Mit der operativen Paranoia, zu der Tantivy Mucker-Maffick Slothrop rät, oder mit Salvador Dali gesprochen: mit der paranoisch-kritischen Aktivität, empfiehlt der Text dazu sogar ein konkretes Verfahren. Allerdings scheint es in der PynchonForschung diesbezüglich zu Missverständnissen gekommen zu sein: Gemeinhin gilt Pynchon als >Autor der Paranoia und tatsächlich spielt die Paranoia als Figur in seinen Texten eine wichtige Rolle. Oftmals wird dabei aber übersehen, dass Pynchons Prosa ihrem grundsätzlichen Gestus nach Satire ist. Die Satire aber muss die Gegenstände, die sie attackiert, zitieren, also: aufgreifen und vorführen. Dabei macht sie sich mit ihnen keineswegs gemein. Ganz im Gegenteil. Deshalb vertrete ich die Auffassung, dass Pynchons Prosa entschieden anti-paranoisch - übrigens auch anti-ödipal (Deleuze und Guattari 1977) - ist. Beides lässt sich am Text gut zeigen. Natürlich grassiert in Gravity’s Rainbow allerorts in der Bevölkerung, im Militär, den Forschungseinrichtungen und den Geheimdiensten - die Paranoia. Man denke allein an die im Text beschriebenen »Achtung! Feind hört mit!«-Papp-Plakate, von denen, als Dachschindeln zweckentfremdet, die immer gleiche »Gestalt mit hochgeschlagenem Mantelkragen und tief ins Gesicht gezogenem Hut « (Pynchon 1981, 678) von oben herab in Erdmanns und Slothrops windschiefes Refugium stiert. Und selbstverständlich internalisiert Slothrop anfangs auch den Verfolgungswahn. Das heißt aber nicht, dass auch die Prosa diese Position übernimmt. Sie ist diesbezüglich viel ambivalenter. 
Greift man die Aphorismen über die Paranoia heraus, die in den Text gestreut werden, könnte man durchaus stutzig werden. Sie lauten:

Sinnsprüche für Paranoiker, 1: Der Meister mag dir verborgen bleiben - doch seine Kreaturen kannst du kitzeln.

(Pynchon 1981, 375)

Sinnsprüche für Paranoiker, 2: In der Arglosigkeit der Kreaturen spiegelt sich die Amoral des Meisters.

(Pynchon 1981, 381)

Sinnsprüche für Paranoiker, 3: Wem es gelingt, dir falsche Fragen einzureden, dem braucht auch vor der Antwort nicht zu bangen

(Pynchon 1981, 397)

Sinnsprüche für Paranoiker, 4: Du versteckst dich, sie suchen.

(Pynchon 1981, 412)

Paranoiker sind Paranoiker nicht etwa (Sinnspruch 5), weil sie paranoisch wären, sondern weil sie sich, verdammte Idioten, andauernd freiwillig in paranoide Situationen begeben.

(Pynchon 1981, 459)

Aus dem Zusammenhang gerissen, erscheinen die Ironiesignale, die die Paranoia als Spiel ausweisen, viel deutlicher. Ähnlich verhält es sich mit dem Ödipus-Komplex. Es steht völlig außer Frage, dass Slothrops Vater Broderick tatsächlich in das »Unternehmen Schwarzknabe« (Pynchon 1981, 448) involviert ist. Als »Schwarzvater« (Pynchon 1981, 449) zeichnet er darin für jene Übereinkunft verantwortlich, nach der Baby Tyrone auf Vermittlung Lyle Blands als Versuchsobjekt an Laszlo Jamfs Psychochemie AG, und damit indirekt an die I.G. Farben, verkauft wurde, wofür diese umgekehrt eine »Zahlungsverpflichtung zugunsten der Harvard-Universität« eingegangen ist, wodurch sie mit einer »Summe von gut 5000 Dollar, die Zinsen eingerechnet« für die dortige Ausbildung Slothrops aufzukommen hatte:

Wenn er's genau bedenkt, hat Slothrop immer Schwierigkeiten gehabt, die dauernden Me-
netekel vom bevorstehenden Familienruin während der ganzen Depression mit dem be-
quemen Studentenleben in Einklang zu bringen, das er in Harvard führte. Tja, und worin
bestand der Handel zwischen seinem Vater und Bland? Ich bin verkauft worden, Jesus
Christus, verkauft worden an die I.G. Farben, wie man ein Stück Rindfleisch verkauft.
Und »Überwachung«? Stinnes hatte, wie jeder Industriemagnat, sein eigenes Spionage-
netz. Genauso die I.G. Soll das heißen, daß Slothrop unter ihrer Beobachtung gestanden
hat, vi-vielleicht seit seiner Geburt? Yaahhh ...
(Pynchon 1981, 449)

An unterschiedlichen Stellen rekurriert der Text auf diesen Teufelspakt und entsprechend wird Slothrop mitunter als »Brodericks und Nallines Schattenkind, ihr nie gebeichteter, ihr Monstersohn« (Pynchon 1981, 1060) bezeichnet - Frankenstein lässt grüßen. Aber damit lässt es der Text nicht bewenden. In einer phantastischen Entgrenzung befreit er die Figur aus ihrer ödipalen Zentrierung, indem er sie im Rahmen einer Prolepse, die in die Zukunft der Raketenstadt reicht, zur Superheldenfigur eines fiktiven Comics stilisiert, in dem Slothrop, 
gemeinsam mit Myrtle Miraculous, Maximilian und dem Schachautomaten Marcel, eine futuristische Truppe bildet. Als die »Verwirrten Vier« (Pynchon 1981, 1057) treten sie »täglich zu ihrem Match gegen den Bösen Paps« (Pynchon 1981, 1058) an, bevor sie merken, „daß die 4 und ihre Vaterverschwörung nicht die ganze Welt sind. Ihr Kampf ist nicht der einzige, ja noch nicht mal der entscheidende« (Pynchon 1981, 1063). So wird letztlich auch der Ödipus-Komplex entgrenzt. Und wie könnte man dieses Verfahren anders beschreiben denn als eine operative oder: kreative, Paranoia? Salvador Dali kennzeichnet sie zunächst als »Hyper-Scharfsinn« (Dali 1973, 11), um dann »die Möglichkeit einer experimentellen Methode ins Auge [zu fassen], die auf dem unmittelbaren Vermögen systematischer, für Paranoia typischer Assoziationen beruht« (Dali 1973, 14), nicht aber mit ihnen identisch ist. Deshalb bemüht er sich auch, möglichst exakt zwischen Paranoia und kritisch-paranoischer Methode $\mathrm{zu}$ differenzieren: »Paranoia: interpretierender Assoziationswahn mit systematischer Struktur - Paranoisch-kritische Aktivität: spontane Methode irrationaler Erkenntnis, die auf der kritisch-interpretierenden Assoziation wahnhafter Phänomene beruht« (Dali 1973, 14) - etwa, indem man den auf die Vaterfigur bezogenen, systematischen Assoziationswahn zum Ausgangspunkt für eine dieses wahnhafte Phänomen in kritischer Absicht mit einer albernen Comic-Story assoziierenden Analyse verbindet und auf diese Weise ad absurdum führt. Dieser Ansatz findet in Gravity's Rainbow auch andernorts seine Entsprechung. Ausgerechnet als »der Regen aufhört«, besinnt sich Slothrop auf das Versprechen, Säure Bummer sein Haschisch zu liefern. In diesem Moment sind ihm »das S-Gerät und das Jamf-Mysterium [...] schon ganz fremd geworden«:

Wenn etwas Tröstliches - Religiöses, wenn man will - in der Paranoia liegt, so gibt es doch auch eine Anti-Paranoia, in der nichts mehr mit irgend etwas anderem verknüpft ist, ein Zustand, den nicht viele von uns lange ertragen. Und genau in diesem Augenblick fühlt Slothrop, wie er in die anti-paranoische Phase seines Kreislaufs hinübergleitet, wie sich die Stadt um ihn zurückzieht, dächerlos, verletzlich, ohne Zentrum wie er selbst, bis nur noch Pappkartonbilder vom Mithörenden Feind zwischen ihm und dem nassen Himmel übrigbleiben.

(Pynchon 1981, 678f.)

So entlädt sich die ursprüngliche Territorialisierung in einer großen, zentrifugalen Bewegung der Deterritorialisierung. Dazu bricht der Text mit nahezu allen gängigen Genrekonventionen der erzählenden Literatur und des Romans, die er der Lächerlichkeit preisgibt. Tradierte Konzepte werden aufgelöst. Typische Ordnungsschemata werden durch Figuren wie das hysteron proteron oder die Inversion verkehrt. Die Grenzen von Sichtbarkeit und Sagbarkeit werden überschritten. Viele generative Subsysteme werden einem amplificatio-Modus unterstellt, in dem der Text die üblichen Maßeinheiten und Gesetzmäßigkeiten der Literatur hemmungslos überbietet. Bekannte Darstellungsformen und Wahrnehmungsweisen werden verfremdet. Verfahren wie der Witz brechen Linearität und Homogenität auf. Der 
Text provoziert mit seiner satirischen Attacke auf das Etablierte und ergeht sich in Exzessen der illegitimen Vermischung, Verknüpfung, Vervielfältigung und Pluralisierung. Als literarischer Kynismus der Postmoderne wendet er sich - im Einklang mit der counter culture seiner Entstehungszeit - gegen den militärisch-industriellen Komplex, gegen die Paranoia und gegen die Ödipalisierung seiner Generation. Mit Nietzsche gesprochen markiert die Kunst der Prosa von Gravity's Rainbow >Die Geburt der Gegenkultur aus dem Geiste des Raketenstaates`.

\section{Literaturverzeichnis}

Bachtin, Michail: Probleme der Poetik Dostoevskijs. Frankfurt a. M., Berlin, Wien 1985.

Dali, Salvador: Die Eroberung des Irrationalen. Essays. Frankfurt a. M., Berlin, Wien 1973.

Debord, Guy: Die Gesellschaft des Spektakels. Berlin 1996.

Deleuze, Gilles und Félix Guattari: Anti-Ödipus. Kapitalismus und Schizophrenie I. Frankfurt a. M. 1977.

Dimeglio, John E.: Vaudeville U.S.A. Bowling Green, OH 1973.

Foucault, Michel: Der Mut zur Wahrheit. Die Regierung des Selbst und der anderen II. Vorlesung am Collège de France 1983/1984. Hrsg. v. Frédéric Gros. Berlin 2010.

Giuriato, Davide, Martin Stingelin und Sandro Zanetti (Hg.): "SCHREIBKUGEL IST EIN DING GLEICH MIR: VON EISEN«. Schreibszenen im Zeitalter der Typoskripte. München 2005.

Giuriato, Davide, Martin Stingelin und Sandro Zanetti (Hg.): „System ohne General«. Schreibszenen im digitalen Zeitalter. München 2006.

Hadot, Pierre: Philosophie als Lebensform. Geistige Übungen in der Antike. Berlin 1991.

Haym, Rudolf: Die romantische Schule. Ein Beitrag zur Geschichte des deutschen Geistes. Berlin 1870.

Jelinek, Elfriede: kein licht am ende des tunnels - nachrichten über thomas pynchon. In: Manuskripte. Zeitschrift für Literatur 52 (1976), 36-44.

Kharpertian, Theodore D.: A Hand to Turn the Time. The Menippean Satires of Thomas Pynchon. Rutherford, Madison, Teaneck und London, Toronto 1990.

Kihss, Peter: Pulitzer Jurors Dismayed on Pynchon. In: The New York Times (8.5.1974), 38.

Kittler, Wolf: Thermodynamik und Guerilla. Zur Methode von Michel Foucaults Archäologie des Wissens. In: Trajekte. Newsletter des Zentrums für Literaturforschung Berlin 2.4 (2002), 16-21.

Kristeva, Julia: Bachtin, das Wort, der Dialog und der Roman. In: Literaturwissenschaft und Linguistik. Ergebnisse und Perspektiven. Bd. 3: Zur linguistischen Basis der Literaturwissenschaft, II. Hrsg. v. Jens Ihwe. Frankfurt a. M. 1972, 345-375.

Lachmann, Tobias: (Literarische) Zerstreuung und Resistenz: Thomas Pynchons »Vineland«. In: Ästhetik und Politik der Zerstreuung. Hrsg v. Tobias Lachmann. Paderborn 2020, 343-385.

Lessing, Gotthold Ephraim: Werke. Vierter Band. Dramatische Schriften. Hrsg. v. Herbert G. Göpfert. München 1973.

Link, Jürgen: Elementare Literatur und generative Diskursanalyse. München 1983.

Link, Jürgen: Normalismus und Antagonismus in der Postmoderne. Krise, New Normal, Populismus. Göttingen 2018. 
Lyotard, Jean-François: La condition postmoderne. Paris 1979.

Matthes, Lothar: Vaudeville. Untersuchungen zu Geschichte und literatursystematischem Ort einer Erfolgsgattung. Heidelberg 1983.

Niehaus, Michael: Die sprechende und die stumme Anekdote. In: Zeitschrift für deutsche Philologie 132.2 (2013), 183-202.

Niehues-Pröbsting, Heinrich: Der Kynismus des Diogenes und der Begriff des Zynismus. Frankfurt a. M. 1988.

Nietzsche, Friedrich: Die Geburt der Tragödie, oder: Griechenthum und Pessimismus. In: ders.: Werke. Kritische Gesamtausgabe. Hrsg. v. Giorgio Colli und Mazzino Montinari et al. Dritte Abteilung, Bd. 1. Berlin, New York 1972, 3-152.

Pethes, Nicolas: Literatur- und Wissenschaftsgeschichte. Ein Forschungsbericht. In: Internationales Archiv für Sozialgeschichte der Literatur 28 (2003), 181-231.

Pynchon, Thomas: Gravity's Rainbow. New York 1973.

Pynchon, Thomas: Die Enden der Parabel. Roman. Reinbek bei Hamburg 1981.

Pynchon, Thomas: Is It O.K. To Be A Luddite? In: The New York Times Book Review 1 (28.10.1984), 40-41.

Pynchon, Thomas: Vorwort. In: ders.: Spätzünder. Frühe Erzählungen. Reinbek bei Hamburg 1985, 9-33.

Pynchon, Thomas: Togetherness. In: Schreibheft. Zeitschrift für Literatur 61 (2003), 13-18.

Schlegel, Friedrich: <ZUR PHILOSOPHIE. b. 1797.> ENDE DER ERSTEN UND UEBERGANG ZUR MORALISCHEN EPOCHE. In: ders.: Kritische Friedrich-Schlegel-Ausgabe. Hrsg. v. Ernst Behler, Jean-Jacques Anstett und Hans Eichner. Bd. 18. München, Paderborn, Wien 1963, 79-117.

Schlegel, Friedrich: Athenäums-Fragmente. In: ders.: Kritische Friedrich-Schlegel-Ausgabe. Hrsg. v. Ernst Behler, Jean-Jacques Anstett und Hans Eichner. Bd. 2. München, Paderborn, Wien 1967, 165-255.

Schlegel, Friedrich: Fragmente zur Litteratur und Poesie. 1797. In: ders.: Kritische Schriften und Fragmente 1794-1818. Studienausgabe. Hrsg. v. Ernst Behler und Hans Eichner.

Bd. 5. Paderborn, München, Wien, Zürich 1988, 184-232.

Siegel, Jules: Who Is Thomas Pynchon ... And Why Did He Take Off With My Wife? In: Playboy 24 (1977), 97, 122, 168-174.

Simon, Ralf: Die Idee der Prosa. Zur Ästhetikgeschichte von Baumgarten bis Hegel mit einem Schwerpunkt bei Jean Paul. München 2013.

Simon, Ralf: Theorie der Prosa. In: Grundthemen der Literaturwissenschaft: Poetik und Poetizität. Hrsg. v. Ralf Simon. Berlin, Boston 2018, 415-429.

Snow, Charles Percy: Die zwei Kulturen. Literarische und naturwissenschaftliche Intelligenz. C. P. Snows These in der Diskussion. Hrsg. v. Helmut Kreuzer. München 1987.

Steinmann, Kurt (Hg.): Das Leben des Diogenes von Sinope erzählt von Diogenes Laertios. Zürich 1999.

Stingelin, Martin: Sturm auf die Informationsmaschinen? In Begleitung von Thomas Pynchons Aufsatz »Is It O.K. to Be a Luddite?«. In: Kanalarbeit. Medienstrategien im Kulturwandel. Hrsg. v. Hans Ulrich Reck. Basel, Frankfurt a. M. 1988, 242-253.

Stingelin, Martin (Hg.): »Mir ekelt vor diesem tintenklecksenden Säkulum«. Schreibszenen im Zeitalter der Manuskripte. München 2004. 
Waddell, Helen: The Wandering Scholars. London 1980.

Weiß, Wolfgang: Satirische Dialogizität und satirische Intertextualität. In: Intertextualität.

Formen, Funktionen, anglistische Fallstudien. Hrsg. v. Ulrich Broich und Manfred Pfister. Tübingen 1985, 244-262.

Wertheim, Arthur Frank: Vaudeville Wars. How the Keith-Albee and Orpheum Circuits Controlled the Big-Time and Its Performers. New York, Basingstoke 2006. 
